\title{
A SOLUTION WITH FREE BOUNDARY FOR NON-NEWTONIAN FLUIDS WITH DRUCKER-PRAGER PLASTICITY CRITERION
}

\author{
E. NTOVORIS ${ }^{1, *}$ And M. ReGis ${ }^{2}$
}

\begin{abstract}
We study a free boundary problem which is motivated by a particular case of the flow of a non-Newtonian fluid, with a pressure depending yield stress given by a Drucker-Prager plasticity criterion. We focus on the steady case and reformulate the equation as a variational problem. The resulting energy has a term with linear growth while we study the problem in an unbounded domain. We derive an Euler-Lagrange equation and prove a comparison principle. We are then able to construct a subsolution and a supersolution which quantify the natural and expected properties of the solution; in particular, we show that the solution has in fact compact support, the boundary of which is the free boundary.

The model describes the flow of a non-Newtonian material on an inclined plane with walls, driven by gravity. We show that there is a critical angle for a non-zero solution to exist. Finally, using the sub/supersolutions we give estimates of the free boundary.
\end{abstract}

Mathematics Subject Classification. 76A05, 49J40, 35R35.

Received November 26, 2016. Accepted June 17, 2018.

\section{INTRODUCTION}

Setting of the problem. We study non-negative solutions $u(y, z)$ of the equation

$$
\left\{\begin{array}{l}
\operatorname{div}(\nabla u+|z| q)=-\lambda \quad \text { in }(-1,1) \times(-\infty, 0), \\
q \in \partial(|\cdot|)(\nabla u)
\end{array}\right.
$$

with $u( \pm 1, z)=0, q=q(y, z), \lambda \geq 0$ and for a function $f: \mathbb{R}^{N} \rightarrow \mathbb{R}, N \in \mathbb{N}$ we define the subdifferential of $f$ at a point $y \in \mathbb{R}^{N}$ as

$$
(\partial f)(y):=\left\{z \in \mathbb{R}^{N}: f(x)-f(y) \geq z \cdot(x-y) \forall x \in \mathbb{R}^{N}\right\}
$$

Keywords and phrases: Non-Newtonian fluid, Drucker-Prager plasticity, variational inequality, free boundary.

1 Université Paris-Est, 6 et 8 avenue Blaise Pascal Cité Descartes - Champs sur Marne 77455 Marne la Vallée Cedex 2, France.

270 rue du Javelot, 75013 Paris, France.

* Corresponding author: eleftherios.ntovoris@enpc.fr 
The variational formulation of (1.1) consists in minimizing the functional

$$
E_{\lambda}(u):=\int_{\Omega} \frac{|\nabla u|^{2}}{2}+|z||\nabla u|-\lambda u
$$

in the space

$$
\mathcal{X}=\mathcal{X}(\Omega):=\left\{u \in W_{0 L}^{1,2}(\Omega), z \nabla u \in L^{1}\left(\Omega, \mathbb{R}^{2}\right)\right\}
$$

with

$$
W_{0 L}^{1,2}(\Omega):=\left\{u \in W^{1,2}(\Omega): u( \pm 1, \cdot)=0\right\}
$$

$\Omega:=(-1,1) \times(-\infty, 0)$. Note that by Remark 2.1 the functional $E_{\lambda}$ is well defined in $\mathcal{X}$. Before we explain the physical interpretation of the mathematical model, we present some of the particularities of the problem. We then state our main results in Section 2.

Since we study the equation (1.1) in an unbounded domain, the variational problem (1.3) is no longer trivial, because it is not clear if the linear term $-\int_{\Omega} \lambda u$ is lower semicontinuous or if the minimizing sequence obtained by the direct method will have a converging subsequence in $\mathcal{X}$. Using Lemma 3.2, we show that the linear term is lower semicontinuous and the well posedness of the problem is established in Theorem 2.2 (i). Also, despite the fact that the energy $E$ includes a term with linear growth (in the gradient variable), a comparison principle still holds for equation (1.1). Using this comparison principle we construct sub/supersolutions and show that in fact the solution of (1.1) is compactly supported.

For the construction of these barriers we use the "curvature like" equation

$$
-\operatorname{div}(|z| q)=\lambda
$$

which is the first variation of the energy $\int_{\Omega}|z||\nabla u|-\lambda u$, with $q=\frac{\nabla u}{|\nabla u|}$ when $\nabla u \neq 0$ and $|q| \leq 1$; then the vector $\frac{\nabla u}{|\nabla u|}$ is the normal to the level sets of $u$. If we suppose that these level sets are given by $-z=\phi(y)$ we are led to study the first variation of the $1 \mathrm{D}$ functional

$$
\int_{-1}^{1}-\phi(y) \sqrt{1+\left|\phi^{\prime}(y)\right|^{2}}+\lambda \phi
$$

We then use the minimizers of (1.6) to be level sets of the barrier functions, the values of which are chosen so that we can control the sign and absolute value of their Laplacian.

Non-Newtonian fluids. The model (1.1) is motivated by the motion of non-Newtonian fluids. Let $\Omega \subset \mathbb{R}^{3}$, open and $v: \Omega \rightarrow \mathbb{R}^{3}$ be the velocity of the fluid, assumed incompressible,

$$
\operatorname{div} v=0
$$

We also assume that the density is constant and by choosing the unit of specific mass, we may take it to be equal to 1 . Let $f: \Omega \rightarrow \mathbb{R}^{3}$ be the external force, then the relevant equation reads as

$$
\operatorname{div} \sigma+f=(\nabla \cdot v) v+\partial_{t} v
$$


where $\sigma$ is the stress tensor and using the usual summation convention we write $(\nabla \cdot v) v=\left(v_{j} \partial_{j} v_{i}\right)_{1 \leq i \leq 3}$. Let $\sigma_{\text {dev }}$ be the stress deviator defined by $\operatorname{tr}\left(\sigma_{\text {dev }}\right)=0$ and

$$
\sigma_{\mathrm{dev}}:=\sigma+p I
$$

where $p$ is the pressure and $I$ is the unit matrix.

We are interested in the flow of rigid visco-plastic fluids, which unlike Newtonian fluids can sustain shear stress. The stress tensor in this case is characterized by a flow/no flow condition, namely when the stress tensor belongs to a certain convex set the fluid behaves like a rigid body, whereas outside this set the material flows

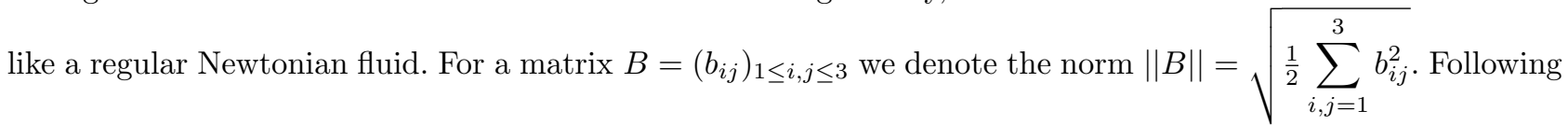
[12] and [5], we limit our discussion to a subclass of (incompressible) visco-plastic fluids characterized by

$$
\begin{cases}\sigma_{\mathrm{dev}}=2 \nu D(v)+k(p) \frac{D(v)}{\|D(v)\|} & \text { if } D(v) \neq 0 \\ \left\|\sigma_{\mathrm{dev}}\right\| \leq k(p) & \text { if } D(v)=0\end{cases}
$$

where the viscosity $\nu>0$ is constant, $k(p)$ is the pressure-dependent yield stress and $D(v):=\left(\nabla v+(\nabla v)^{T}\right) / 2$. The above constitutive laws is a result of a superposition of the viscous contribution $2 \nu D(v)$ and a contribution related to plasticity effects $k(p) \frac{D(v)}{\|D(v)\|}$, which is independent of the norm of the strain rate $\|D(v)\|$. For constant yield limit $k(p)$ we retrieve the regular Bingham model, which is a generalized Newtonian problem, i.e. the constitutive laws in this case are described by a dissipative potential, see [6], [8], chapter 3 and references therein. In this paper, we will assume the Drucker-Prager plasticity criterion

$$
k(p):=\mu_{s} p,
$$

where $\mu_{s}:=\tan \delta_{s}$, with $\delta_{s}$ the internal friction (static) angle. The existence of a dissipative potential in the case of Bingham flows allows for a variational formulation of the problem which guarantees its well-posedness; for quasi-static Bingham flows see for example [8]. The case of a Drucker-Prager criterion, however, falls in a wider class of constitutive laws called " $\mu(I)$-rheology" which are in general ill-posed, see [1, 24]. The strong geophysical interest in the model (1.11) supports however our study. A main result of the present work is that for one-directional steady flows the model is well-posed.

Flow in one direction. We study the well-posedness and certain quantitative properties of quasi-static solutions of (1.7)-(1.8), (1.10)-(1.11), for a material which flows on an inclined plane with sidewalls. Physically, the walls are relevant to the flow of a material in a channel. We assume that the inclination angle is constant $\theta$ and the material moves only in the direction $x$ under the effect of gravity, see Figure 1a. In what follows, we will assume that the velocity field is of the form $v(x, y, z)=(u(y, z), 0,0)$ for $(x, y, z) \in\{(x, y, z): 0 \leq x,-l \leq$ $y \leq l,-A \leq z \leq h(y)\}$ where $A>0, h(y)$ is the interface separating the fluid and the air and $z=-A$ is the surface of the inclined plane, the width of which is equal to $2 l$, Figure $1 \mathrm{a}$.

We are interested in the properties of the material as we increase the inclination angle $\theta$. We call solid and liquid phases the sets $\{(y, z): u(y, z)=0\}$ and $\{(y, z): u(y, z)>0\}$, respectively (often abbreviated as $\{u=0\},\{u>0\}$ resp.), while their common boundary is the relevant free boundary. We note that usually in the literature the set separating the two phases is considered to be $\partial\{\nabla u \neq 0\}$, but approximating this set would require different methods and more regularity of the solution. However, there is still one more free boundary remaining, i.e. the curve that separates the solid from the liquid phase.

For $A$ small we expect that for a sufficiently large angle $\theta$ all of the material will move due to the gravity, namely there is no solid phase. Whereas, if $A$ is large enough, even if the inclination is large we expect that there will be a solid phase. In order to study the behavior and shape of the liquid/solid phases as we increase 


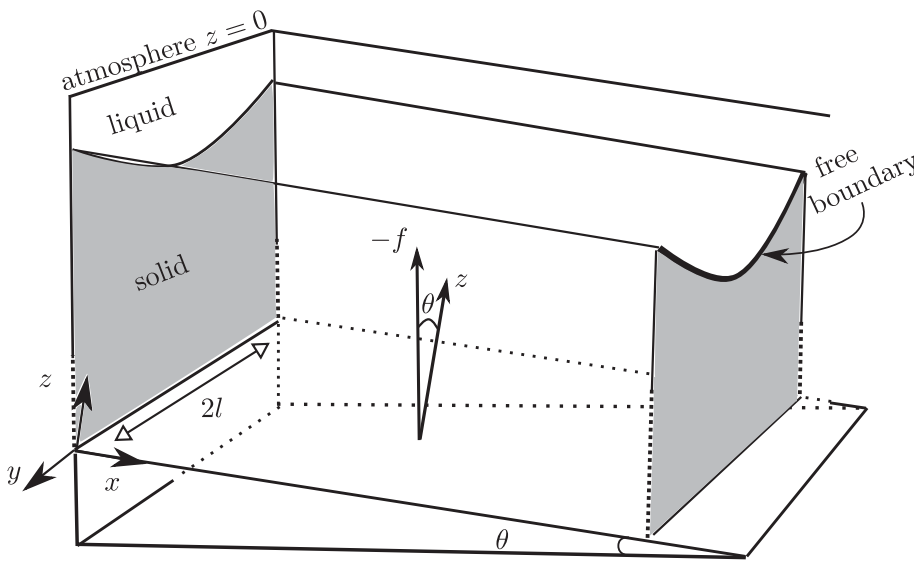

(a) Steady flow on an inclined plane.

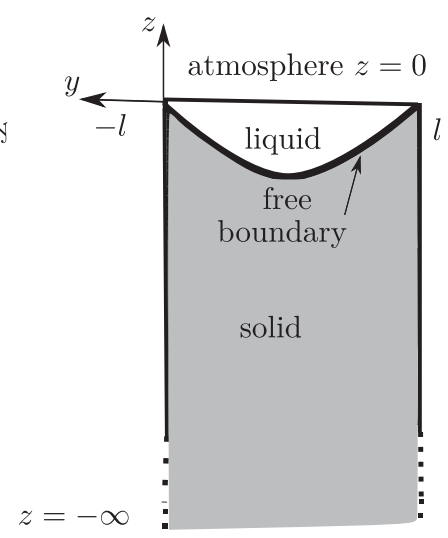

(b) 2-D cross section

Figure 1. Flow on an inclined plane.

the inclination angle, we consider the case where $-A=-\infty$. In this case, since there is no bottom plane, the angle $\theta$ is the angle between the force $-f$ and the (positive) $z$-axis. The axes $x, y, z$, and the vector $f$ remain as they were when $A$ was finite. As we will see later in Theorem 2.3 the function $u$ has actually compact support. We can now consider again the problem where the distance of the bottom plane from the atmosphere is finite, with zero boundary conditions at the bottom plane, Proposition 2.5. The solution of this problem is actually independent of the depth of the bottom plane, as long as the plane is below the boundary of the support of the solution $u$ which was considered in the unbounded domain. The problem in the unbounded domain, Figure 1b, is no longer trivial.

By the form of $v$, the incompressibility condition (1.7) is trivially satisfied and equation (1.8) with $\partial_{t} v=0$ becomes

$$
\operatorname{div} \sigma_{\mathrm{dev}}=-f+\nabla p
$$

with $\sigma_{\text {dev }}$ given by (1.10) and

$$
f=\left(g_{0} \sin \theta, 0,-g_{0} \cos \theta\right),
$$

with $g_{0}$ the gravity acceleration. We also assume that $p=p(y, z)$. We calculate

$$
D(v)=\frac{1}{2}\left(\begin{array}{ccc}
0 & \partial_{y} u & \partial_{z} u \\
\partial_{y} u & 0 & 0 \\
\partial_{z} u & 0 & 0
\end{array}\right)
$$

and $\|D u\|=\frac{1}{2}|\nabla u|$, with $\nabla u=\left(\partial_{y} u, \partial_{z} u\right)$. If we substitute (1.14) in (1.10), equation (1.12) become, for $D(v) \neq 0$ or equivalently $\nabla u \neq 0$,

$$
\begin{aligned}
\nu \operatorname{div}(\nabla u)+\mu_{s} \operatorname{div}\left(p \frac{\nabla u}{|\nabla u|}\right) & =-g_{0} \sin \theta \\
0 & =\partial_{y} p \\
0 & =g_{0} \cos \theta+\partial_{z} p
\end{aligned}
$$


where the divergence is taken for the coordinates $(y, z)$. If we integrate equation (1.17) from $z$ to $h(y)$ we get $p(y, z)=(h(y)-z) g_{0} \cos \theta$, since $p(y, h(y))=0$ near the atmosphere. But because of equation (1.16) and because $\theta \in\left[0, \frac{\pi}{2}\right)$ we have $h(y)=h \equiv$ constant. For simplicity we take $h=0$; then the pressure is given by

$$
p(y, z)=|z| g_{0} \cos \theta
$$

We are led to study the following equation

$$
\left\{\begin{array}{l}
\nu \operatorname{div}(\nabla u)+\mu_{s} g_{0} \cos \theta \operatorname{div}(|z| q)=-g_{0} \sin \theta \quad \text { in }(-l, l) \times(-\infty, 0), \\
q \in \partial(|\cdot|)(\nabla u),
\end{array}\right.
$$

where $\partial(|\cdot|)$ is the subdifferential of the absolute value. If $(u, q)$ is such that (1.19) holds, with $q=q(y, z)=$ $\left(q_{1}(y, z), q_{2}(y, z)\right)$, then $|q| \leq 1$ and $q=\frac{\nabla u}{|\nabla u|}$ for $\nabla u \neq 0$. Then by the definition of $\sigma_{\mathrm{dev}}$ and (1.10) we have

$$
\sigma_{\mathrm{dev}}=\nu\left(\begin{array}{ccc}
0 & \partial_{y} u & \partial_{z} u \\
\partial_{y} u & 0 & 0 \\
\partial_{z} u & 0 & 0
\end{array}\right)+\mu_{s}|z| g_{0} \cos \theta\left(\begin{array}{ccc}
0 & q_{1} & q_{2} \\
q_{1} & 0 & 0 \\
q_{2} & 0 & 0
\end{array}\right)
$$

with $v(x, y, z)=(u(y, z), 0,0)$. Moreover, $\sigma_{\text {dev }}$ solves equation (1.12) with $f$ given by (1.13) and $p$ by (1.18).

Boundary conditions for (1.19). On the surface of the material $z=0$ we assume a no stress condition, i.e. $\sigma \cdot(0,0,1)=0$; since the pressure is zero on the surface near the atmosphere, this condition becomes $\sigma_{\mathrm{dev}} \cdot(0,0,1)=0$, with $\sigma_{\mathrm{dev}}$ as in $(1.20)$. Then the stress free condition becomes (since $z=0$ )

$$
\partial_{z} u(y, 0)=0 .
$$

For simplicity, on the lateral boundary $y= \pm 1$ we assume the Dirichlet conditions $u=0$ (no slip). As mentioned in [13], no-slip conditions can be achieved by making the sidewalls rough by gluing one layer of beads on them.

Variational formulation. The variational formulation of equation (1.19) with boundary conditions (1.21) and the homogeneous Dirichlet conditions on the lateral boundary constitutes in minimizing the energy

$$
\int_{(-l, l) \times(-\infty, 0)} \nu \frac{|\nabla u|^{2}}{2}+\mu_{s}|z| g_{0} \cos \theta|\nabla u|-\left(g_{0} \sin \theta\right) u
$$

with zero lateral boundary conditions, i.e. $u( \pm 1, \cdot)=0$. Despite the fact that the energy $(1.22)$ is convex, the unboundedness of the domain makes the application of the direct method non-trivial.

Let $\tilde{u}$ be a solution of (1.19)-(1.21), in order to simplify further the equation (1.19) we set

$$
u(y, z)=\frac{\nu}{\mu_{s} g_{0} \cos \theta} \frac{\tilde{u}(l y, l z)}{l^{2}}, \quad(y, z) \in(-1,1) \times(-\infty, 0)
$$

we also define

$$
\lambda:=\frac{\tan \theta}{\mu_{s}},
$$


then $\partial|\cdot|(\nabla u(y, z))=\partial|\cdot|(\nabla \tilde{u}(l y, l z))$ and therefore, $u$ given by (1.23) solves the equation (1.1) if and only if $\tilde{u}$ solves (1.19). In Theorem 2.3 we show that the minimizer has compact support, something which is not clear since the domain is unbounded. We also show that the critical angle for an non-zero minimizer to exist is $\arctan \mu_{s}$. Namely, for $\theta>\arctan \mu_{s}$ there exists a non-zero solution with a free boundary separating the two phases, while for $0 \leq \theta \leq \arctan \mu_{s}$ the solution is zero. The time dependent, one dimensional analogue of our case is studied in [4]; the authors prove that for $\theta>\arctan \mu_{s}$ there is no solution with solid phase while in our case the solution always has a solid phase. The difference of course lies in our two dimensional setting of the problem in which the existence of the walls where the velocity vanishes is crucial, not just for the physical relevance of the problem. Indeed since we study minimizers of (1.3) in an unbounded domain we will often need to apply Poincaré's inequality, for this reason we need that the projection of the domain in one of the coordinate axis is bounded. In [19] the authors also prove that for $\theta \leq \arctan \mu_{s}$ the flowing material stops moving in finite time.

Review of the literature. For an extensive review of non-Newtonian fluids see [6], also [8] and references therein and [21] for evolutionary problems. The flow of a viscoplastic material with " $\mu(I)-$ rheology" is relatively new in the literature, see for example [12].

In [16], the author studies a more general form of the Bingham problem, with a $p$-Dirichlet energy. He develops a similar theory of subsolutions and supersolutions as we do and proves a comparison principle. Then he identifies the critical value of $\lambda$ in the functional $\int_{\Omega} \frac{1}{p}|\nabla u|^{p}+|\nabla u|-\lambda u$, when the integral is computed over a bounded domain with $C^{1}$-boundary, with the Cheeger constant of this domain. The author also observes the natural formation of flat regions, i.e. regions with positive measure where the solution is constant. Properties of these flat regions are also studied in [15]. It is in these flat regions that the non-local character of singular equations like (1.1) is observed, because the ratio $\nabla u /|\nabla u|$ is not defined. Other singular equations with similar non-local character have been studied in [11] and have applications in crystal growth problems. In the same paper the authors also develop a viscosity theory for these equations and prove a comparison principle.

The inviscid case, i.e. for $\nu=0$ is similar to another scalar model with applications in image processing, the total variation flow, see for example $[2,25]$ and for a viscosity solution approach in [10]. Although the total variation bears more similarities with the Bingham case, many of the tools used to study our problem are similar. In fact the total variation is more difficult to study because of the lack of the quadratic term in the energy which leads to lack of regularity of the solution. For the inviscid case our energy (1.3) falls into a wider class, the "total variation functionals" see [3], Hypothesis 4.1. We refer to [20] for simulations of a regularized Drucker-Prager model with application to granular collapse. Concerning the case of the inclined plane see $[13,22]$.

Organization of the paper. In Section 2, we state our main results, Theorems 2.2 and 2.3. We also give Proposition 2.5 which states the connection between the problems which are considered in an unbounded and a bounded domain. In Section 3.1 we study the 1-dimensional analogue of (1.3) which we use in Lemma 3.2 in Section 3.2; this lemma is the crucial step in order to prove that the linear term $-\lambda \int_{\Omega} u$ is lower semicontinuous. The proof of Theorem 2.2 (i) is given at the end of Section 3.2. In Section 3.3 we study an approximate problem of the minimizer of (1.3) which helps us to prove certain regularity properties of the solution. Using the approximate minimizer we can also calculate the first variation of (1.3). We then prove Theorem 2.2 (ii)-(iv) at the end of Section 3.3. Finally, in Lemma 4.4 we construct a solution of (1.5) which we use together with the comparison principle from Section 4.1, in Sections 4.3 and 4.4 in order to construct a subsolution and a supersolution respectively. The proof of the second main Theorem 2.3 is again split into two parts; the lower bound is given in Section 4.3 while the upper bound at the end of Section 4.4. The Figures 2-6 have been made with Mathematica. 


\section{MAIN RESULTS}

We begin with a technical remark.

Remark 2.1. We have $\mathcal{X}(\Omega) \subset W^{1,1}(\Omega)$, which justifies the choice of the space $\mathcal{X}$ as a natural functional space for the functional (1.3). Indeed,

$$
\int_{\{|z| \geq 1\} \cap \Omega}|\nabla u| \leq \int_{\{|z| \geq 1\} \cap \Omega}|z||\nabla u|<\infty
$$

from which get that $u \in L^{1}(\Omega)$ by Poincaré's inequality, see [18], Theorem 12.17; note also that in our case the proof of Poincaré's inequality requires only that elements of the space $W^{1,2}(\Omega)$ are zero on the lateral boundary of $\Omega(i . e$. on $\{ \pm 1\} \times(0, \infty))$. In fact, since the width of the walls is 2 we have $\int_{\Omega}|u|^{p} \leq \frac{2^{p}}{p} \int_{\Omega}|\nabla u|^{p}$, for $p=1,2$.

Let

$$
\Lambda:=\left\{q: q \in L_{l o c}^{2}\left(\Omega, \mathbb{R}^{2}\right),|q| \leq 1 \text { a.e. }\right\} .
$$

Let $\hat{\Omega}:=(-1,1) \times \mathbb{R}, u \in W_{0 L}^{1,2}(\Omega)$, we denote by $\hat{u} \in W_{0 L}^{1,2}(\hat{\Omega})$ the reflection of $u$ with respect to the $y$-axis, i.e.

$$
\hat{u}(y, z):= \begin{cases}u(y, z) & \text { if }(y, z) \in \Omega, \\ u(y,-z) & \text { if } y, z) \in \hat{\Omega} \backslash \Omega .\end{cases}
$$

Throughout the paper we will denote the space $\mathcal{X}(\Omega)$ simply by $\mathcal{X}$. Only in Lemma 3.3 we will use the explicit notation, this time for the space $\mathcal{X}(\hat{\Omega})$. The weak formulation of (1.1) is

$$
\begin{cases}\int_{\Omega} \nu \nabla u \cdot \nabla \varphi+|z| q \cdot \nabla \varphi=\lambda \int_{\Omega} \varphi & \text { for all } \varphi \in \mathcal{X} \\ q \cdot \nabla u=|\nabla u| & \text { a.e. }\end{cases}
$$

for some $\lambda \geq 0, q \in \Lambda$. We can now state our first main theorem.

Theorem 2.2. (Existence and uniqueness of minimizers of (1.3))

Let $\lambda \geq 0$ and $E_{\lambda}$ be given by (1.3). Then

(i) there exists a unique $0 \leq u_{\lambda} \in \mathcal{X}$ such that

$$
E_{\lambda}\left(u_{\lambda}\right)=\inf _{v \in \mathcal{X}} E_{\lambda}(v)
$$

moreover, $u_{\lambda} \equiv 0$ if $\lambda \in[0,1]$ and $u_{\lambda} \neq \equiv 0$ if $\lambda \in(1,+\infty)$,

(ii) there exists $q \in \Lambda$ such that $\left(u_{\lambda}, q\right)$ solves (2.3),

(iii) $u_{\lambda} \in C_{\mathrm{loc}}^{0, \alpha}(\Omega)$ for all $\alpha \in(0,1)$, in fact $\hat{u}_{\lambda} \in W_{\mathrm{loc}}^{2,2}(\hat{\Omega})$ and $\partial_{z} u_{\lambda}(y, 0)=0$ for $y \in(-1,1)$,

(iv) if $\lambda>1$, the pair $\left(u_{\lambda}, q\right)$ obtained in (ii) is unique in the sense that if $\left(\bar{u}_{\lambda}, \bar{q}\right) \in \mathcal{X} \times \Lambda$ is another pair satisfying (2.3) then

$$
u=\bar{u} \quad \text { in } \Omega, \quad \text { and } \quad q=\bar{q} \text {, a.e. in }\{\nabla u \neq 0\} .
$$

We set

$$
I:=\inf _{v \in \mathcal{X}} E_{\lambda}(v)
$$


Note that by the continuity of the non-negative function $u_{\lambda}$ in Theorem 2.2 we can define the free boundary as the common boundary $\Omega \cap \partial\left\{u_{\lambda}>0\right\}=\Omega \cap \partial\left\{u_{\lambda}=0\right\}$. Moreover, the critical value $\lambda=1$ in the previous theorem is also a critical value of the physical solution by (1.23), (1.24) and it does not depend on the viscosity constant $\nu$ or the width of the walls.

We will give some notations in order to present our second result; the motivation for these notations will become clear in the proofs of the relevant propositions. Let $\lambda>1$, for $Z \in\left[\frac{1}{\lambda}, \frac{1}{\lambda-1}\right]$ we define

$$
f_{\lambda}(Z):=\frac{1}{\left(\lambda^{2}-1\right)^{3 / 2}}\left\{\operatorname{Arcsin}\left[\left(\lambda^{2}-1\right) Z-\lambda\right]-\lambda \sqrt{1-\left(\left(\lambda^{2}-1\right) Z-\lambda\right)^{2}}\right\} .
$$

As we will see in the proof of Lemma 4.4, the function $f_{\lambda}$ is strictly increasing in the interval $\left[\frac{1}{\lambda}, \frac{1}{\lambda-1}\right]$; we can therefore define the following function

$$
\phi_{K, \lambda}(y):=K(\lambda) f_{\lambda}^{-1}\left(f_{\lambda}\left(\frac{1}{\lambda-1}\right)+\frac{|y|}{K(\lambda)}\right) \quad y \in[-1,1]
$$

where

$$
K(\lambda):=\frac{1}{f_{\lambda}\left(\frac{1}{\lambda}\right)-f_{\lambda}\left(\frac{1}{\lambda-1}\right)} .
$$

Note that by the monotonicity of $f_{\lambda}$ we have $K(\lambda)<0$. We also define the half cone

$$
\mathcal{C}_{\lambda}:=\left\{(y, z) \in \mathbb{R}^{2}: 0<|y|<z \frac{\lambda}{K(\lambda)}\right\}
$$

and

$$
\operatorname{Epi}^{\smile}(\lambda):=\left\{(y, z) \in \Omega: z>\phi_{K, \lambda}(y)\right\} .
$$

In Lemma 4.4 we show that the sets in $(2.9)$ are increasing in $\lambda$ in the sense that $\operatorname{Epi}^{\smile}(\lambda) \subsetneq \operatorname{Epi}^{\smile}(\bar{\lambda})$ for $\bar{\lambda}>\lambda$, see Figure 3a. For $\lambda_{1}>\lambda$ we set

$$
\begin{gathered}
\vartheta_{\lambda, \lambda_{1}}:=\frac{\lambda_{1}-\lambda}{2\left(1+\left(\frac{\lambda_{1}}{K\left(\lambda_{1}\right)}\right)^{2}\right)}, \\
b\left(\lambda, \lambda_{1}\right):=1+\sqrt{\frac{\lambda_{1}}{2 \vartheta_{\lambda, \lambda_{1}}}}, \\
\Pi\left(\lambda, \lambda_{1}\right):=\frac{-K\left(\lambda_{1}\right)}{\lambda_{1}-1} b\left(\lambda, \lambda_{1}\right)+\frac{K(\lambda)}{\lambda-1},
\end{gathered}
$$

and

$$
\operatorname{Epi}_{\smile}\left(\lambda_{1}\right):=\left\{(y, z) \in \Omega: z>b\left(\lambda, \lambda_{1}\right) \phi_{K, \lambda_{1}}\left(\frac{y}{b\left(\lambda, \lambda_{1}\right)}\right)\right\}
$$


In Lemma 4.4 we see that $\min _{|y| \leq 1} \phi_{K, \lambda}(y)=\phi_{K, \lambda}(0)=\frac{K(\lambda)}{\lambda-1}$ for all $\lambda>1$, and therefore, the function $\Pi$ in $(2.12)$ is the distance between the projections on the $z$-axis of the epigraphs $\operatorname{Epi}^{\smile}(\lambda)$ and $\Omega \backslash \operatorname{Epi}_{\triangleleft}\left(\lambda_{1}\right)$, respectively. Using (2.7) we calculate

$$
\frac{K\left(\lambda_{1}\right)}{\lambda_{1}-1}=\frac{2\left(\lambda_{1}+1\right) \sqrt{\lambda_{1}^{2}-1}}{2 \sqrt{\lambda_{1}^{2}-1}+\pi+2 \operatorname{Arcsin}\left(\frac{1}{\lambda_{1}}\right)},
$$

then $\lim _{\lambda_{1} \rightarrow+\infty} \frac{K\left(\lambda_{1}\right)}{\lambda_{1}-1}=+\infty$, and similarly one can see that $\lim _{\lambda_{1} \rightarrow+\infty} \frac{K\left(\lambda_{1}\right)}{\lambda_{1}}=+\infty$; if we combine the above two limits, one can check that for all $\lambda>1$,

$$
\lim _{\lambda_{1} \rightarrow+\infty} \Pi\left(\lambda, \lambda_{1}\right)=+\infty
$$

We also have

$$
\lim _{\lambda_{1} \rightarrow \lambda} \Pi\left(\lambda, \lambda_{1}\right)=+\infty
$$

If we combine (2.14), (2.15) and the fact that $\Pi$ is continuous we get that for every fixed $\lambda>1$ the function $\Pi(\lambda, \cdot)$ attains a minimum for some $\lambda_{1}^{\star}>\lambda$. In fact, numerical simulations (see Fig. 6a) suggest the function $\Pi(\lambda, \cdot)$ attains the minimum at a unique $\lambda_{1}^{\star}>\lambda$, but the analytical calculations are too complicated to check.

In the following theorem we gather the main properties of the solution obtained in Theorem 2.2.

Theorem 2.3. (The support of the minimizer)

Let $\lambda>1, \mathcal{X}$ be defined in (1.4), $\left(u_{\lambda}, q\right) \in \mathcal{X} \times \Lambda$ be a solution of (2.3), $\lambda_{1}>\lambda$ and Epi $\backsim\left(\lambda_{1}\right)$ be as in (2.13). Then the function $u_{\lambda}$ has compact support and its support can be estimated as follows

$$
\operatorname{Epi}^{\smile}(\lambda) \subset \operatorname{supp} u_{\lambda} \subset \operatorname{Epi}_{\smile}\left(\lambda_{1}\right) .
$$

Moreover, we can optimize estimate (2.16) by choosing $\lambda_{1}=\lambda_{1}^{\star}$.

Remark 2.4. (Consequences of Theorem 2.3)

1. In Lemma 4.4 , we show that the function $\phi_{K, \lambda}$ has a strictly negative maximum, therefore, estimate (2.16) implies that the free boundary $\partial\left\{u_{\lambda}>0\right\}$ never reaches the surface of the atmosphere $\{z=0\}$.

2. Notice that the sets $\operatorname{Epi}^{\smile}(\lambda)$ and $\operatorname{Epi}_{\smile}\left(\lambda_{1}\right)$ can also estimate the support of the physical solution, by (1.23) and they are independent of the viscosity $\nu$.

3. Since the minimizer in Theorem 2.2 is studied in the half stripe $\Omega$, the regularity which is stated in the same theorem holds up to the free boundary.

Using the fact that the minimizer of (1.3) in $\mathcal{X}$ has compact support, we can get some results for the problem of a material flowing on an inclined plane with finite distance from the atmosphere. Let

$$
E_{\lambda}^{A}(u)=\int_{\Omega_{A}} \frac{|\nabla u|^{2}}{2}+|z||\nabla u|-\lambda u
$$

where $\Omega_{A}=(-1,1) \times(-A, 0), A>0$. The following proposition shows that the minimizer of $(2.17)$ in the space

$$
\tilde{\mathcal{X}}_{A}:=\left\{u \in W^{1,2}(\Omega): u( \pm 1, \cdot)=0, u(\cdot,-A)=0\right\},
$$


coincides with the minimizer of (1.3) in $\mathcal{X}$, and does not depend on $A$ as long as $A$ is large enough.

Proposition 2.5. (The case of the finite bottom)

Let $u_{\lambda}, u_{\lambda, A}$ be the minimizers of $E_{\lambda}$ and $E_{\lambda}^{A}$ in the spaces $\mathcal{X}$ and $\tilde{\mathcal{X}}_{A}$ respectively. Then if $A$ is large enough, for example $A>b\left(\lambda, \lambda_{1}^{*}\right) \frac{K(\lambda)}{\lambda-1}$, with $\lambda_{1}^{*}$ as in Theorem 2.3, we have $u_{\lambda}=u_{\lambda, A}$, in $\Omega_{A}$.

The existence of the minimizer $u_{\lambda, A}$ is straightforward, since the domain $\Omega_{A}$ is bounded. As for the equality of the two minimizers, it can be easily proved using similar arguments as in Step 3 of the proof of Theorem 2.2 (i) and the fact that $u_{\lambda}(\cdot, z)=0$ for $z \geq A$, for $A$ large. We can choose the plane $z=A$ to be outside the support of $u_{\lambda}$, i.e. $A>b\left(\lambda, \lambda_{1}^{*}\right) \min _{|y| \leq 1} \phi_{K, \lambda}(y)=b\left(\lambda, \lambda_{1}^{*}\right) \frac{K(\lambda)}{\lambda-1}$.

\section{Existence AND Uniqueness of Minimizers of (1.3)}

\subsection{A cross section variational problem}

Let $A>0$ and for $w \in W_{0}^{1,2}(-1,1)$ we consider the energy

$$
\epsilon_{A}(w):=\int_{-1}^{1}\left(\frac{\left|w^{\prime}(y)\right|^{2}}{2}+A\left|w^{\prime}(y)\right|\right) \mathrm{d} y
$$

Using the direct method of calculus of variations it is not difficult to show that for $A>0$ and $m>0$ there exists a unique function $w$ solving

$$
\epsilon_{A}(w)=I_{m}^{A}
$$

where

$$
I_{m}^{A}:=\inf _{\substack{\bar{w} \in W_{0}^{1,2}(-1,1) \\ \int_{-1}^{1} \bar{w}=m}} \epsilon_{A}(\bar{w}) .
$$

The uniqueness of the minimizer of $\epsilon_{A}$ follows by the strict convexity of the functional or by using similar arguments as in the proof of Step 3 of Theorem 2.2 (i).

We define the set theoretic sign function as

$$
\operatorname{sign}(r):= \begin{cases}\left\{\frac{r}{|r|}\right\} & \text { if } r \neq 0 \\ {[-1,1]} & \text { else. }\end{cases}
$$

Proposition 3.1. (Characterization of the minimizer of $\epsilon_{A}(w)$ )

Let $A, m>0, \lambda_{A, m}$ be the non-negative root in $[A+m,+\infty)$ of

$$
\begin{gathered}
2 \lambda_{A, m}^{3}-3 \lambda_{A, m}^{2}(A+m)+A^{3}=0, \\
a:=\frac{A}{\lambda_{A, m}}<1,
\end{gathered}
$$




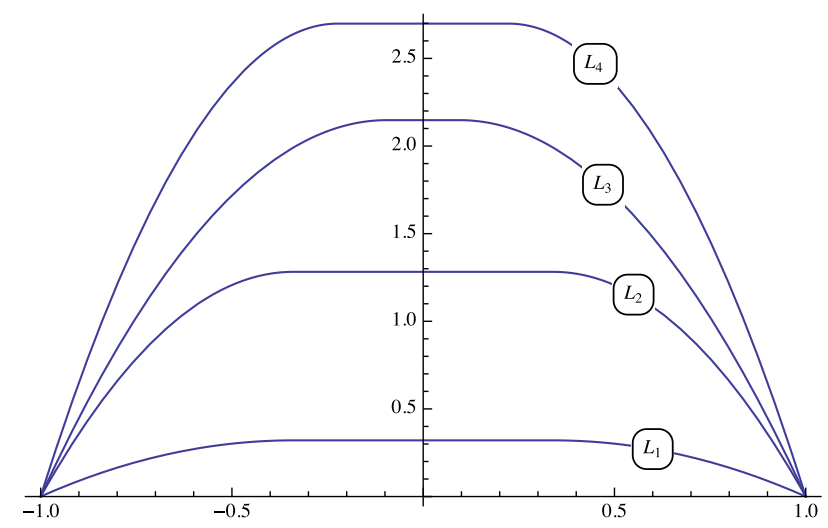

Figure 2. $L_{i}, i=1,2,3,4$ are the graph of $w$ for $(m, A)=(0.5,0.5),(2,2),(3,0.5),(4,2)$, respectively.

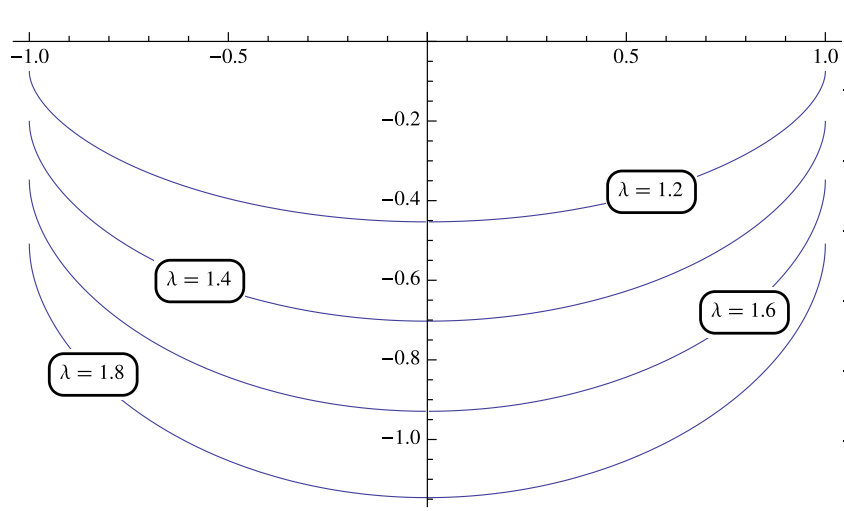

(a) $\phi_{K, \lambda}$ for $\lambda=1.2,1.4,1.6,1.8$.

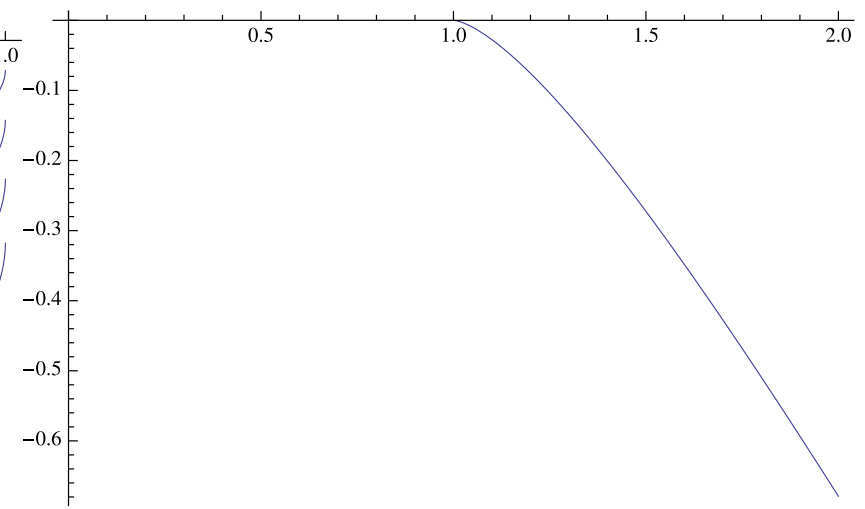

(b) $\phi_{K, \lambda}(1)$

Figure 3. Parametrized solutions.

$$
w(y):= \begin{cases}A\left(-\frac{y^{2}}{2 a}+|y|+\frac{1}{2 a}-1\right) & a<|y|<1, \\ A \frac{(a-1)^{2}}{2 a} & |y| \leq a,\end{cases}
$$

and

$$
q(y):= \begin{cases}-\frac{y}{|y|} & a<|y|<1, \\ -\frac{y}{a} & |y| \leq a,\end{cases}
$$

Then $\left(w, q, \lambda_{A, m}\right)$ solves the equation

$$
-w^{\prime \prime}(y)-A(q(y))^{\prime}=\lambda_{A, m}, \quad \text { for a.e. } y \in(-1,1),
$$

and $\int_{-1}^{1} w=m$. In particular, $w$ is the unique minimizer of (3.1) corresponding to the volume constraint $m$. 
Proof of Proposition 3.1

A simple calculation shows that the trinomial (3.3) has a unique non-negative root $\lambda_{A, m} \geq A+m$. For a.e. $y \in(-1,1)$ we have

$$
w^{\prime \prime}(y)= \begin{cases}-\frac{A}{a} & a<|y|<1, \\ 0 & |y|<a\end{cases}
$$

and

$$
q^{\prime}(y)= \begin{cases}0 & a<|y|<1 \\ -\frac{1}{a} & |y|<a\end{cases}
$$

Using (3.7), (3.8) and (3.4) we deduce that $\left(w, q, \lambda_{A, m}\right)$ solves (3.6).

A simple calculation together with equations (3.4) and (3.3) imply that

$$
\int_{-1}^{1} w=\frac{A}{3 a}(1-a)^{2}(2+a)=\frac{\left(\lambda_{A, m}-A\right)^{2}\left(2 \lambda_{A}+A\right)}{3 \lambda_{A}^{2}}=m .
$$

It remains to show that $w$ is the minimizer of $\epsilon_{A}$ in $W_{0}^{1,2}(-1,1)$ which corresponds to the constraint $m$. First we notice that $q(x) \in \operatorname{sign}\left(w^{\prime}(x)\right)=\partial(|\cdot|)\left(w^{\prime}(x)\right)$ for $x \in(-1,1)$. Let $v \in W_{0}^{1,2}(-1,1)$ with $\int_{-1}^{1} v=m$. It is

$$
\begin{aligned}
\epsilon_{A}(v)-\epsilon_{A}(w) & \geq \int_{-1}^{1} w^{\prime}(v-w)^{\prime}+A q(v-w)^{\prime} \\
& \geq-\int_{-1}^{1}\left(w^{\prime \prime}+A q^{\prime}\right)(v-w)=\lambda_{A, m} \int_{-1}^{1}(v-w)=0
\end{aligned}
$$

where in the last equation we used (3.6).

\subsection{The direct method for $E_{\lambda}$}

The lower semicontinuity of the term $-\int_{\Omega} \lambda u$ in (1.3) under the weak topology of $W^{1,2}$ is not trivial since the integral is not evaluated in a bounded domain. The following lemma shows that the $L^{1}$-tails of a sequence of functions will converge to zero if the respective values of the functional $E_{\lambda}$ are uniformly bounded.

Lemma 3.2. (Compensation of the mass)

Let $\left\{v_{k}\right\}_{k \in \mathbb{N}} \subset \mathcal{X}$, and suppose that there exists a non-negative constant $c$ independent of $k$ such that $E_{\lambda}\left(v_{k}\right)<c$ for all $k \in \mathbb{N}$. Then

$$
\lim _{l \rightarrow+\infty}\left(\sup _{k}\left(\int_{l}^{+\infty} \int_{-1}^{1} v_{k}(y,-A) \mathrm{d} y \mathrm{~d} A\right)\right)=0 .
$$

Proof of Lemma 3.2

Step 1: An estimate for the minimum of $\epsilon_{A}$.

Let $A>0$, and define

$$
m_{A}^{k}:=\int_{-1}^{1} v_{k}(y,-A) \mathrm{d} y
$$


Let $\epsilon_{A}$ be given by (3.1) and $I_{m_{A}^{k}}^{A}$ be the minimum of $\epsilon_{A}$ corresponding to the constraint $m_{A}^{k}$. Let $\lambda_{A, m_{A}^{k}}$ be the root of (3.3); then

$$
m_{A}^{k}=\frac{\left(\lambda_{A, m_{A}^{k}}-A\right)^{2}\left(2 \lambda_{A}+A\right)}{3 \lambda_{A}^{2}} .
$$

Using (3.5) we calculate

$$
I_{m_{A}^{k}}^{A}=\frac{A^{2}}{a}\left(\frac{2}{3} \frac{(1-a)^{3}}{a}+(1-a)^{2}\right)=\frac{\left(\lambda_{A, m_{A}^{k}}-A\right)^{2}\left(2 \lambda_{A}+A\right)}{3 \lambda_{A}}=m_{A}^{k} \lambda_{A, m_{A}^{k}} .
$$

By Proposition 3.1 we have $\lambda_{A, m_{A}^{k}} \geq A+m_{A}^{k}$, hence equation (3.12) becomes

$$
I_{m_{A}^{k}}^{A} \geq m_{A}^{k}\left(A+m_{A}^{k}\right) \geq A m_{A}^{k} .
$$

Step 2: The tails of $v_{k}$ converge uniformly to 0 .

We argue by contradiction, suppose that

$$
\sup _{k}\left(\int_{l}^{+\infty} m_{A}^{k} \mathrm{~d} A\right) \nrightarrow 0 \text { as } l \rightarrow+\infty
$$

then there are $\varepsilon>0$ and a sequence $l_{j} \rightarrow+\infty$ as $j \rightarrow+\infty$ such that

$$
\sup _{k}\left(\int_{l_{j}}^{+\infty} m_{A}^{k} \mathrm{~d} A\right) \geq \varepsilon \text {. }
$$

By Fubini's lemma we have for $l_{j}>\lambda$

$$
\begin{aligned}
E_{\lambda}\left(v_{k}\right) & =\int_{0}^{+\infty} \int_{-1}^{1} \frac{\left|\nabla v_{k}\right|^{2}}{2}+A\left|\nabla v_{k}\right|-\lambda v_{k} \mathrm{~d} y \mathrm{~d} A \\
& \geq \int_{0}^{+\infty} \int_{-1}^{1} \frac{\left|\partial_{y} v_{k}\right|^{2}}{2}+A\left|\partial_{y} v_{k}\right|-\lambda v_{k} \mathrm{~d} y \mathrm{~d} A \\
& \geq \int_{l_{j}}^{+\infty} I_{m_{A}^{k}}^{A}-\lambda m_{A}^{k} \mathrm{~d} A \geq \int_{l_{j}}^{+\infty} m_{A}^{k}\left(l_{j}-\lambda\right) \mathrm{d} A,
\end{aligned}
$$

where in the last inequality we used (3.11), (3.2) and (3.13). Taking the supremum over $k \in \mathbb{N}$ we get, using (3.14)

$$
c \geq \sup _{k} E_{\lambda}\left(v_{k}\right) \geq \sup _{k}\left(\int_{l_{j}}^{+\infty} m_{A}^{k}\left(l_{j}-\lambda\right) \mathrm{d} A\right) \geq\left(l_{j}-\lambda\right) \varepsilon \rightarrow+\infty \quad \text { as } l_{j} \rightarrow+\infty,
$$

a contradiction.

We have the following lemma.

Lemma 3.3. (Approximation by smooth functions)

Let $v \in \mathcal{X}(\hat{\Omega})$. Then there is a sequence $v_{A} \in W_{0}^{1,2}(\hat{\Omega})$ with compact support in $\bar{\Omega}$ such that

$$
v_{A} \rightarrow v \text { in } W^{1,2}(\hat{\Omega}) \cap L^{1}(\hat{\Omega})
$$


and

$$
\lim _{A \rightarrow+\infty} \int_{\hat{\Omega}}|z|\left|\nabla v_{A}-\nabla v\right|=0
$$

Proof of Lemma 3.3

First we note that $v \in L^{1}(\hat{\Omega})$ by Remark 2.1. Let $A>1$, we define the cut off functions $\eta_{A} \in W^{1, \infty}(\mathbb{R})$ by

$$
\eta_{A}(z):= \begin{cases}1 & \text { if }|z| \leq A, \\ 1-\frac{1}{A}(|z|-A) & \text { if } A \leq|z| \leq 2 A, \\ 0 & \text { if } 2 A \leq|z| .\end{cases}
$$

Then

$$
\left|\eta_{A}^{\prime}(z)\right| \leq \frac{2}{|z|} \text { a.e. }
$$

The functions $v_{A}(y, z):=\eta_{A}(z) v(y, z)$ belong to $W^{1,2}(\hat{\Omega})$, they have compact support in $\bar{\Omega}_{A}$, with $\hat{\Omega}_{A}:=$ $(-1,1) \times(-2 A, 2 A)$ and zero trace on $\partial \hat{\Omega}_{A}$. Since the boundary of each $\hat{\Omega}_{A}$ is Lipschitz and bounded we have by [18], Theorem 15.29 that $v_{A} \in W_{0}^{1,2}\left(\hat{\Omega}_{A}\right)$. It is not difficult to see that $v_{A} \rightarrow v$ in $W^{1,2}(\hat{\Omega})$. We will show that $\lim _{A \rightarrow+\infty}|z|\left|\nabla v_{A}-\nabla v\right|=0$.

By (3.18) we have

$$
\begin{aligned}
\int_{\hat{\Omega}}|z|\left|\nabla v_{A}-\nabla v\right| & \leq \int_{\hat{\Omega}}|z|\left(\left|\eta_{A}^{\prime} v\right|+\left|\eta_{A}-1\right||\nabla v|\right) \\
& \leq \int_{\hat{\Omega} \cap\{A<|z|<2 A\}} 2|v|+\int_{\hat{\Omega} \cap\{A<|z|\}}|z||\nabla v|
\end{aligned}
$$

then using the fact that $|z||\nabla v|,|v| \in L^{1}(\hat{\Omega})$ the right hand side of the above estimate converges to zero as $A \rightarrow+\infty$.

The convergence in $L^{1}(\hat{\Omega})$ in (3.16) follows by Remark 2.1.

For two sets $U, U^{\prime} \subset \mathbb{R}^{2}$, by $U \subset \subset U^{\prime}$ we mean that $U$ is relatively compact in $U^{\prime}$, i.e. $\bar{U} \subset U^{\prime}$ and $\bar{U}$ is compact. Also, for a function $u(y, z)$ we define the positive part $u^{+}(y, z):=\max \{u(y, z), 0\}$.

\section{Proof of Theorem 2.2 (i)}

Step 1. The minimizing sequence.

We focus on the case where $\lambda>0$, since for $\lambda=0$ the minimizer of $E_{\lambda}$ is trivially the zero function. We fix $\lambda>0$. Let $u \in \mathcal{X}$, using Poincaré's inequality in $\Omega$ (Remark 2.1) we get

$$
\begin{aligned}
E_{\lambda}(u) & =\int_{\Omega} \frac{|\nabla u|^{2}}{2}+|z||\nabla u|-\lambda \int_{\Omega} u \\
& \geq \int_{\Omega} \frac{|\nabla u|^{2}}{2}+(|z|-2 \lambda)|\nabla u| .
\end{aligned}
$$

We split the last integral in the domains $\{|z| \geq 2 \lambda\} \cap \Omega$ and $\{|z| \leq 2 \lambda\} \cap \Omega$ and get

$$
\begin{aligned}
E_{\lambda}(u) & \geq \int_{\{|z| \leq 2 \lambda\} \cap \Omega} \frac{|\nabla u|^{2}}{2}-2 \lambda|\nabla u| \\
& \geq \int_{\{|z| \leq 2 \lambda\} \cap \Omega}-2 \lambda^{2}>-\infty .
\end{aligned}
$$


We may now find $u_{k} \in \mathcal{X}$ with $\lim _{k \rightarrow+\infty} E_{\lambda}\left(u_{k}\right)=\inf _{v \in \mathcal{X}} E_{\lambda}(v)$. We will denote by $c$ a generic positive constant which does not depend on the parameter $k$. There is a positive constant $c$ such that $\sup _{k \in \mathbb{N}} E_{\lambda}\left(u_{k}\right) \leq c$. Then as before we use Poincare's inequality to get

$$
\begin{aligned}
c & \geq \int_{\{|z| \leq 2 \lambda\} \cap \Omega} \frac{\left|\nabla u_{k}\right|^{2}}{2}+(|z|-2 \lambda)\left|\nabla u_{k}\right|+\int_{\{|z| \geq 2 \lambda\} \cap \Omega} \frac{\left|\nabla u_{k}\right|^{2}}{2} \\
& \geq \int_{\{|z| \leq 2 \lambda\} \cap \Omega} \frac{\left|\nabla u_{k}\right|^{2}}{2}-\frac{\left|\nabla u_{k}\right|^{2}}{4}-(|z|-2 \lambda)^{2}+\int_{\{|z| \geq 2 \lambda\} \cap \Omega} \frac{\left|\nabla u_{k}\right|^{2}}{2},
\end{aligned}
$$

where in the second inequality we used Young's inequality $\left(|a||b| \leq \frac{b^{2}}{4}+a^{2}\right)$. It is easy now to see that

$$
\int_{\Omega}\left|\nabla u_{k}\right|^{2} \leq c
$$

Then by Poincare's inequality and compactness there is $u \in W_{0 L}^{1,2}(\Omega)$ such that $u_{k} \rightarrow u$ up to a subsequence as $k \rightarrow+\infty$.

Using similar arguments we get $c \geq \int_{\Omega}(|z|-2 \lambda)\left|\nabla u_{k}\right|$, while by splitting the integral in the domains $\{|z| \geq$ $4 \lambda\} \cap \Omega=\{|z|-2 \lambda \geq|z| / 2\} \cap \Omega$ and $\{|z| \leq 4 \lambda\} \cap \Omega$ we get

$$
\frac{1}{2} \int_{\{|z|-2 \lambda \geq|z| / 2\} \cap \Omega}|z|\left|\nabla u_{k}\right| \leq \int_{\{|z| \geq 4 \lambda\} \cap \Omega}(|z|-2 \lambda)\left|\nabla u_{k}\right| \leq c-\int_{\{|z| \leq 2 \lambda\} \cap \Omega}(|z|-2 \lambda)\left|\nabla u_{k}\right|,
$$

since $\int_{\{2 \lambda \leq|z| \leq 4 \lambda\} \cap \Omega}(|z|-2 \lambda)\left|\nabla u_{k}\right| \geq 0$. We can now bound the right hand side of (3.20) using Hölder's inequality and (3.19) and get eventually that $\int_{\{|z| \geq 4 \lambda\} \cap \Omega}|z|\left|\nabla u_{k}\right| \leq c$. Using Hölder's inequality and (3.19), one can also bound the quantity $\int_{\{|z| \leq 4 \lambda\} \cap \Omega}|z|\left|\nabla u_{k}\right|$ uniformly in $k$. We can therefore conclude that

$$
\int_{\Omega}|z|\left|\nabla u_{k}\right| \leq c
$$

where again $c$ is a positive constant independent of $k$.

Step 2. Lower semicontinuity.

We will show that

$$
\int_{\Omega} \frac{|\nabla u|^{2}}{2}+|z||\nabla u| \leq \liminf _{k \rightarrow+\infty} \int_{\Omega} \frac{\left|\nabla u_{k}\right|^{2}}{2}+|z|\left|\nabla u_{k}\right|
$$

and

$$
-\lambda \int_{\Omega} u \leq \liminf _{k \rightarrow+\infty}\left(-\lambda \int_{\Omega} u_{k}\right)
$$

Equations (3.19), (3.21) and (3.22) imply that $u \in \mathcal{X}$ and then $u \in L^{1}(\Omega)$ by Remark 2.1. Also, equations (3.22) and (3.23) together imply that $E_{\lambda}(u) \leq \liminf _{k \rightarrow+\infty} E_{\lambda}\left(u_{k}\right)$, which shows that $u$ is a minimizer of $E_{\lambda}$ in $\mathcal{X}$. Since the integrand in (3.22) is non-negative, convex in the gradient variable and measurable in the $z$ variable, the inequality (3.22) follows from [9], Chapter I, Theorem 2.5. 
For $l>0$ fixed, we have

$$
\begin{aligned}
\int_{\Omega} u_{k} & =\int_{l}^{+\infty} \int_{-1}^{1} u_{k}(y,-A) \mathrm{d} y \mathrm{~d} A+\int_{0}^{l} \int_{-1}^{1} u_{k}(y,-A) \mathrm{d} y \mathrm{~d} A \\
& \leq \sup _{k}\left(\int_{l}^{+\infty} \int_{-1}^{1} u_{k}(y,-A) \mathrm{d} y \mathrm{~d} A\right)+\int_{0}^{l} \int_{-1}^{1} u_{k}(y,-A) \mathrm{d} y \mathrm{~d} A .
\end{aligned}
$$

Since $E_{\lambda}\left(u_{k}\right)$ is uniformly bounded we can apply Lemma 3.2 and get that (3.10) holds for the sequence $u_{k}$. Using (3.10) and the fact that $u \in L^{1}(\Omega)$, we can take the limsup in (3.24), as $k \rightarrow+\infty$ and then $l \rightarrow+\infty$ and get $\limsup _{k \rightarrow+\infty} \int_{\Omega} u_{k} \leq \int_{\Omega} u$, i.e. (3.23). This completes the proof of the lower semi-continuity of $E_{\lambda}$ and hence the existence of a minimizer $u \in \mathcal{X}$.

Step 3. Uniqueness of a non-negative minimizer.

Let $u, \tilde{u} \in \mathcal{X}$ be two minimizers, then using similar arguments as in [6], Section 3.5.4, page 36 one can show that

$$
\begin{aligned}
& \int_{\Omega} \nabla u \cdot(\nabla \tilde{u}-\nabla u)+\int_{\Omega}|z||\nabla \tilde{u}|-\int_{\Omega}|z||\nabla u| \geq \lambda \int_{\Omega} \tilde{u}-u \\
& \int_{\Omega} \nabla \tilde{u} \cdot(\nabla u-\nabla \tilde{u})+\int_{\Omega}|z||\nabla u|-\int_{\Omega}|z||\nabla \tilde{u}| \geq \lambda \int_{\Omega} u-\tilde{u} .
\end{aligned}
$$

If we add equations (3.26) and (3.27) we get

$$
\int_{\Omega}|\nabla u-\nabla \tilde{u}|^{2} \leq 0
$$

hence $u=\tilde{u}$ in $\Omega$, since they also have the same lateral boundary conditions.

We have by [26], Corollary 2.1.8, page 47, that $\nabla u^{+}=(\nabla u) \cdot \chi_{\{u>0\}}$, where by $\chi_{\{u>0\}}$ we denote the characteristic function of the set $\{(y, z): u(y, z)>0\}$. Since also $-\lambda \int_{\Omega} u^{+} \leq-\lambda \int_{\Omega} u$ we have $E_{\lambda}\left(u^{+}\right) \leq E_{\lambda}(u)$, hence $u=u^{+}$by the uniqueness of minimizers.

Step 4. Case $\lambda \in[0,1]$.

In this step we assume that $\lambda \in[0,1]$. Our goal is to show that

$$
E_{\lambda}(u) \geq 0, \quad \text { for all } u \in \mathcal{X},
$$

then because $0 \in \mathcal{X}$ and $E_{\lambda}(0)=0$ we get that the unique minimizer of $E_{\lambda}$ is the zero function. In view of Lemma 3.3, it is enough to prove (3.28) for functions $u$ with $\hat{u} \in W_{0}^{1,2}(\hat{\Omega})$ and have compact support in $\overline{\hat{\Omega}}$. Let $u$ be such a function, then as in Step 3 we have

$$
E_{\lambda}\left(u^{+}\right) \leq E_{\lambda}(u)
$$

Suppose that the compact support of $\hat{u}^{+}$is contained in $[-1,1] \times(-A, A)$ where $A$ is large enough, then we have 


$$
\begin{aligned}
\int_{\Omega}|z|\left|\nabla u^{+}\right| & \geq \int_{-1}^{1} \int_{0}^{A}|z|\left|\frac{\partial u^{+}}{\partial z}\right| \mathrm{d} z \mathrm{~d} y \\
& \geq\left|\int_{-1}^{1} \int_{0}^{A} z \frac{\partial u^{+}}{\partial z} \mathrm{~d} z \mathrm{~d} y\right| \\
& =\int_{\Omega} u^{+}
\end{aligned}
$$

in the last equality we used integration by parts. This estimate together with (3.29) and the fact that $\lambda \leq 1$ gives

$$
E_{\lambda}(u) \geq E_{\lambda}\left(u^{+}\right) \geq(1-\lambda) \int_{\Omega} u^{+} \geq 0
$$

Step 5. Case $\lambda \in(1,+\infty)$.

In this step we assume that $\lambda \in(1,+\infty)$. Our goal is to prove that there is $u \in \mathcal{X}$ with $E_{\lambda}(u)<0$. Let $\varphi \in C^{\infty}(-1,1), \varphi \geq 0$ with $\varphi(-1)=0=\varphi(1)$ and $\int_{-1}^{1} \varphi=1$ (for example $\varphi(y)=\frac{3}{4}\left(1-y^{2}\right)$ ). We define

$$
u(y, z):=k^{-3} e^{k z} \varphi(y),
$$

where $k>0$ is large enough, to be chosen later. We have $u \in \mathcal{X}$ and

$$
\begin{aligned}
\int_{\Omega} \frac{|\nabla u|^{2}}{2} & =\frac{1}{2} \int_{-1}^{1}\left[k^{-2}\left(\varphi^{\prime}(y)\right)^{2}+\varphi^{2}(y)\right] \mathrm{d} y \int_{-\infty}^{0}\left(k^{-2} e^{k z}\right)^{2} \mathrm{~d} z \\
& =\frac{A_{k}}{4} k^{-5}
\end{aligned}
$$

where we set $A_{k}:=\int_{-1}^{1}\left[k^{-2}\left(\varphi^{\prime}(y)\right)^{2}+\varphi^{2}(y)\right] \mathrm{d} y$. Also

$$
\begin{aligned}
\int_{\Omega}|z||\nabla u| & =\int_{-1}^{1} \sqrt{k^{-2}\left(\varphi^{\prime}(y)\right)^{2}+\varphi^{2}(y)} \mathrm{d} y \int_{-\infty}^{0}|z| k^{-2} e^{k z} \mathrm{~d} z \\
& =B_{k} \int_{-\infty}^{0}|z| k^{-2} e^{k z} \mathrm{~d} z
\end{aligned}
$$

where $B_{k}:=\int_{-1}^{1} \sqrt{k^{-2}\left(\varphi^{\prime}(y)\right)^{2}+\varphi^{2}(y)} \mathrm{d} y$. If we integrate by parts the second product component of the right hand side of (3.31) we get

$$
\int_{-\infty}^{0}|z| k^{-2} e^{k z} \mathrm{~d} z=\int_{-\infty}^{0} k^{-3} e^{k z}=\int_{-\infty}^{0} k^{-3} e^{k z} \int_{-1}^{1} \varphi=\int_{\Omega} u
$$

then (3.31) becomes

$$
\int_{\Omega}|z||\nabla u|=B_{k} \int_{\Omega} u
$$

We also have $\int_{\Omega} u=k^{-4}$, then we can write $E_{\lambda}(u)$ using (3.30) and (3.32) as

$$
E_{\lambda}(u)=\frac{A_{k}}{4} k^{-5}+\left(B_{k}-\lambda\right) k^{-4} .
$$


Next we note that $B_{k} \geq 1$, is decreasing in $k$ (and so is $A_{k}$ ) and $B_{k} \rightarrow 1$ as $k \rightarrow+\infty$. Since $\lambda>1$ we can find $k_{0}$ large enough such that $B_{k_{0}}<\lambda$, then (3.33) becomes

$$
E_{\lambda}(u) \leq \frac{A_{k_{0}}}{4} k^{-5}+\left(B_{k_{0}}-\lambda\right) k^{-4}
$$

for all $k \geq k_{0}$. We can now conclude if we choose $k \geq k_{0}$ large enough, since the function $k^{-5}$ decreases faster than $k^{-4}$, for example $k>\max \left\{k_{0}, \frac{A_{k_{0}}}{4\left(\lambda-B_{k_{0}}\right)}\right\}$.

\subsection{Approximation of $E_{\lambda}$ by a smooth energy}

Let $\lambda>0, u_{\lambda}$ be the minimizer of $E_{\lambda}$ given by Theorem 2.2 (i). For $A>0$ we define $\hat{\Omega}_{A}:=\{(y, z) \in \hat{\Omega}$ : $|z| \leq A\}, \Omega_{A}:=\Omega \cap \hat{\Omega}_{A}$ and

$$
\mathcal{H}_{A}:=\left\{v \in W^{1,2}\left(\Omega_{A}\right), v=u_{\lambda}, \text { on } \partial \Omega_{A} \backslash\{z=0\}\right\},
$$

We are interested in approximate minimizers of (1.3), for this we study the minimizers in $\mathcal{H}_{A}$ of the approximate functional

$$
E_{\varepsilon, \lambda}^{A}(u):=\int_{\Omega_{A}} \frac{|\nabla u|^{2}}{2}+|z| \sqrt{\varepsilon^{2}+|\nabla u|^{2}}-\lambda u
$$

where $\varepsilon>0$.

Since we have mixed boundary conditions, an easy way to describe the space of test functions for the weak formulation of the first variation of $(3.34)$ is to use reflection in the domain $\hat{\Omega}_{A}$. We will simply write $\hat{\varphi} \in$ $W_{0}^{1,2}\left(\hat{\Omega}_{A}\right)$ for the test functions. We have the following proposition.

Proposition 3.4. ( $W_{\text {loc }}^{2,2}$ regularity of approximate problem)

Let $A, \varepsilon, \lambda>0$. Then there exists a unique minimizer $u_{\varepsilon, A} \in \mathcal{H}_{A}$ of $E_{\varepsilon, \lambda}^{A}$. Moreover, $\hat{u}_{\varepsilon, A} \in W_{\mathrm{loc}}^{2,2}\left(\hat{\Omega}_{A}\right)$ and the following equation holds

$$
\int_{\Omega_{A}} \nabla u_{\varepsilon, A} \cdot \nabla \varphi+|z| \frac{\nabla u_{\varepsilon, A} \cdot \nabla \varphi}{\sqrt{\varepsilon^{2}+\left|\nabla u_{\varepsilon, A}\right|^{2}}}=\lambda \int_{\Omega_{A}} \varphi, \quad \text { for all } \hat{\varphi} \in W_{0}^{1,2}\left(\hat{\Omega}_{A}\right),
$$

and $\partial_{z} u_{\varepsilon, A}(y, 0)=0$ for $y \in(-1,1)$.

The existence of a minimizer is a consequence of the direct method in the bounded domain $\Omega_{A}$, while the regularity results are standard. We give a sketch of the Proof of Proposition 3.4 in Appendix A.

Proof of Theorem 2.2 (ii)-(iv)

Step 1. Solutions of the E-L equation are minimizers of (1.3).

First we will show that for any pair $(u, q) \in \mathcal{X} \times \Lambda$ which satisfies equation (2.3), $u$ is a minimizer of $E_{\lambda}$. Let $v \in \mathcal{X}$, using (2.3) and the fact that $|q| \leq 1$ it is easy to check that $q \in \partial|\cdot|(\nabla u)$ in $\Omega$. By the definition of the subdifferential we have

$$
E_{\lambda}(v)-E_{\lambda}(u) \geq \int_{\Omega} \nabla u \cdot \nabla(v-u)+|z| q \cdot \nabla(v-u)-\lambda \int_{\Omega}(v-u)=0
$$

where we used (2.3) with test function $\varphi=v-u \in \mathcal{X}$. 
Step 2. Approximating solutions.

As usual we will focus in the case $\lambda>0$. Let $u=u_{\lambda}$ be the minimizer of $E_{\lambda}$ given by Theorem 2.2 (i). For $\varepsilon>0$ let $u_{\varepsilon, A}$ be the minimizer of $E_{\varepsilon, \lambda}^{A}$ given by Proposition 3.4, then for all $A>0$ we will show that $u_{\varepsilon, A} \rightarrow u$ strongly as $\varepsilon \rightarrow 0$, in $W^{1,2}\left(\Omega_{A}\right)$ up to a subsequence. Extending $u_{\varepsilon, A}$ by $u_{\lambda}$ outside $\Omega_{A}$, we can write the following variational inequalities as in the Step 3 of the proof of Theorem 2.2 (i)

$$
\int_{\Omega_{A}} \nabla u \cdot\left(\nabla u_{\varepsilon, A}-\nabla u\right)+\int_{\Omega_{A}}|z|\left|\nabla u_{\varepsilon, A}\right|-\int_{\Omega_{A}}|z||\nabla u| \geq \lambda \int_{\Omega_{A}} u_{\varepsilon, A}-u
$$

and

$$
\int_{\Omega_{A}} \nabla u_{\varepsilon, A} \cdot\left(\nabla u-\nabla u_{\varepsilon, A}\right)+\int_{\Omega_{A}}|z| \sqrt{\varepsilon^{2}+|\nabla u|^{2}}-\int_{\Omega_{A}}|z| \sqrt{\varepsilon^{2}+\left|\nabla u_{\varepsilon, A}\right|^{2}} \geq \lambda \int_{\Omega_{A}} u-u_{\varepsilon, A} .
$$

Adding inequalities (3.37) and (3.38), we get

$$
\begin{aligned}
\int_{\Omega_{A}}\left|\nabla u_{\varepsilon, A}-\nabla u\right|^{2} & \leq \int_{\Omega_{A}}|z|\left(\left|\nabla u_{\varepsilon, A}\right|-\sqrt{\varepsilon^{2}+\left|\nabla u_{\varepsilon, A}\right|^{2}}\right)+|z|\left(\sqrt{\varepsilon^{2}+|\nabla u|^{2}}-|\nabla u|\right) \\
& \leq \int_{\Omega_{A}}|z|\left(\sqrt{\varepsilon^{2}+|\nabla u|^{2}}-|\nabla u|\right) \\
& =\int_{\Omega_{A}}|z| \frac{\varepsilon^{2}}{\sqrt{\varepsilon^{2}+|\nabla u|^{2}}+|\nabla u|} \leq A\left|\Omega_{A}\right| \varepsilon .
\end{aligned}
$$

Then using also Poincare's inequality we get for all $A>0$ and up to a subsequence

$$
\nabla u_{\varepsilon, A} \rightarrow \nabla u, u_{\varepsilon, A} \rightarrow \text { u a.e. in } \Omega_{A} \text { as } \varepsilon \rightarrow 0 .
$$

Also, for $q_{\varepsilon, A}:=\frac{\nabla u_{\varepsilon, A}}{\sqrt{\varepsilon^{2}+\left|\nabla u_{\varepsilon, A}\right|^{2}}}$ we have $q_{\varepsilon, A} \cdot \nabla u_{\varepsilon, A} \leq\left|\nabla u_{\varepsilon, A}\right|$. Then using (3.39) it is not difficult to see that

$$
q_{\varepsilon, A} \cdot \nabla u_{\varepsilon, A} \rightarrow|\nabla u| \text { a.e. in } \Omega_{A} \text { as } \varepsilon \rightarrow 0 .
$$

Since $q_{\varepsilon, A} \in L^{2}\left(\Omega_{A}, \mathbb{R}^{2}\right)$ with $\left|q_{\varepsilon, A}\right| \leq 1$, there exists $q_{A} \in L^{2}\left(\Omega_{A}, \mathbb{R}^{2}\right)$ with $\left|q_{A}\right| \leq 1$ and such that $q_{\varepsilon, A}$ converges weakly to $q_{A}$ as $\varepsilon \rightarrow 0$. Then using also (3.39) we have $\lim _{\varepsilon \rightarrow 0} \int_{U} q_{\varepsilon, A} \cdot \nabla u_{\varepsilon, A}=\int_{U} q_{A} \cdot \nabla u$ for all $U \subset \subset \Omega_{A}$ and by (3.40) we get that $q_{A} \cdot \nabla u=|\nabla u|$ a.e. in $\Omega_{A}$. Extending $q_{A}$ by zero outside $\Omega_{A}$ we may write $q_{A} \in L_{\text {loc }}^{2}\left(\Omega, \mathbb{R}^{2}\right)$. As before we can find $q \in L_{\text {loc }}^{2}\left(\Omega, \mathbb{R}^{2}\right)$, with $|q| \leq 1$, such that $q_{A}$ converges weakly to $q$ in $L_{\text {loc }}^{2}\left(\Omega, \mathbb{R}^{2}\right)$, as $A \rightarrow+\infty$, hence $q \cdot \nabla u=|\nabla u|$ a.e.

Step 3. Passing to the limit $\varepsilon \rightarrow 0, A \rightarrow+\infty$.

Let $\varphi$ with $\hat{\varphi} \in W_{0}^{1,2}(\hat{\Omega})$ and compact support in $\hat{\Omega}$. Then equation (3.35) with $A$ large enough holds for this test function and since $q_{\varepsilon, A}$ is bounded we can pass to the limit as $\varepsilon \rightarrow 0$ and get

$$
\int_{\Omega} \nabla u \cdot \nabla \varphi+|z| q_{A} \cdot \nabla \varphi=\lambda \int_{\Omega} \varphi
$$

We can now pass to the limit as $A \rightarrow+\infty$ and using also Lemma 3.3 we get (2.3). 
Step 4. Uniqueness.

Let $(u, q),(\bar{u}, \bar{q})$ be two solutions of $(2.3)$ then by Step 1 we have $u=\bar{u}$, since minimizers of (1.3) in $\mathcal{X}$ are unique by Theorem 2.2 (i). In the set $\{\nabla u \neq 0\}$ the vectors $q, \bar{q}$ are parallel to $\nabla u$ and so is $q-\bar{q}$, but since $(q-\bar{q}) \cdot \nabla u=0$ by $(2.3)$ we have $q=\bar{q}$ a.e. in $\{\nabla u \neq 0\}$.

Step 5. Neumann condition.

We denote by $\partial_{x_{i}}, i=1,2$ respectively the derivatives $\partial_{y}, \partial_{z}$. Let $i, j \in\{1,2\}, \hat{U} \subset \subset \hat{\Omega}_{A}$. By Proposition 3.4 we have that $\hat{u}_{\varepsilon, A} \in W_{\mathrm{loc}}^{2,2}(\hat{U})$, by Lemma A.1 the second derivatives of $\hat{u}_{\varepsilon, A}$ are uniformly bounded in $L^{2}(\hat{U})$, hence for $\varphi \in W_{0}^{1,2}(\hat{U})$ we have (up to a subsequence)

$$
\int_{\hat{U}} \partial_{x_{i}} \hat{u} \partial_{x_{j}} \varphi=\lim _{\varepsilon \rightarrow 0} \int_{\hat{U}} \partial_{x_{i}} \hat{u}_{\varepsilon, A} \partial_{x_{j}} \varphi=-\lim _{\varepsilon \rightarrow 0} \int_{\hat{U}} \partial_{x_{j}} \partial_{x_{i}} \hat{u}_{\varepsilon, A} \varphi=-\int_{\hat{U}} g \varphi
$$

for some function $g \in L^{2}(\hat{U})$. We have proved that $\hat{u} \in W_{\mathrm{loc}}^{2,2}(\hat{\Omega})$, then applying a Sobolev embedding theorem ([7], Sect. 5.6.3) we get that $\hat{u} \in C_{\text {loc }}^{0, \alpha}(\hat{\Omega})$ for all $\alpha \in(0,1)$. As in the proof of Proposition 3.4 we can now define the trace of the derivative of $u$ on $\{z=0\}$ and $\partial_{z} u(y, 0)=0$ for $y \in(-1,1)$.

\section{Properties of the SOLUtion}

\subsection{Comparison Principle}

In view of Theorem 2.2 (i) we will assume that $\lambda>1$ for the rest of the paper.

Definition 4.1. Sub/supersolution.

Let $u \in \mathcal{X}$ be a non-negative function and $q \in \Lambda$, with $\Lambda$ as in (2.1). We call the pair $(u, q)$ a subsolution (resp. a supersolution) of (2.3) if

$$
\begin{cases}\int_{\Omega} \nabla u \cdot \nabla \varphi+|z| q \cdot \nabla \varphi \leq \lambda \int_{\Omega} \varphi\left(\text { resp. } \geq \lambda \int_{\Omega} \varphi\right) & \text { for all } \varphi \in \mathcal{X}, \varphi \geq 0 \\ q \cdot \nabla u=|\nabla u| & \text { a.e. in } \Omega .\end{cases}
$$

Proposition 4.2. Comparison principle.

Let $u, v \in \mathcal{X}, q_{u}, q_{v} \in \Lambda$ with $\left(u, q_{u}\right),\left(v, q_{v}\right)$ a subsolution and a supersolution respectively of $(2.3)$, with $0=u \leq v$ on $\{-1,1\} \times(-\infty, 0)$ in the sense of traces, then

$$
u \leq v, \quad \text { in } \Omega .
$$

Proof of Proposition 4.2

Let $\varphi=(u-v)_{+}$, then $\varphi \in \mathcal{X}$. If we write the inequality in (4.1) for $u$ and $v$ with $\varphi$ as a test function, and subtract one from the other we get

$$
\int_{\Omega} \nabla(u-v) \cdot \nabla(u-v)_{+}+|z|\left(q_{u}-q_{v}\right) \cdot \nabla(u-v)_{+} \leq 0
$$

and if we use [26], Corollary 2.1.8, page 47, we can write it as

$$
\int_{\Omega}|\nabla(u-v)|^{2} \chi_{\{u-v \geq 0\}} \leq-\int_{\Omega}|z|\left[\left(q_{u}-q_{v}\right) \cdot \nabla(u-v)\right] \chi_{\{u-v \geq 0\}} .
$$


Next we calculate, using the properties of $q_{u}, q_{v}$ in Definition 4.1

$$
\begin{aligned}
\left(q_{u}-q_{v}\right) \cdot(\nabla u-\nabla v) & =|\nabla u|-q_{u} \cdot \nabla v-q_{v} \cdot \nabla u+|\nabla v| \\
& \geq|\nabla u|-|\nabla u|+|\nabla v|-|\nabla v|=0, \text { a.e. }
\end{aligned}
$$

then (4.2) implies

$$
\nabla(u-v)=0, \quad \text { a.e. in }\{u-v \geq 0\}
$$

which means $\nabla(u-v)_{+}=0$ almost everywhere. Using the boundary conditions we can conclude that $(u-v)_{+}=0$ and hence $u \leq v$ a.e. in $\Omega$.

Remark 4.3. (Monotonicity in $\lambda$ )

Let $u_{\lambda}$ be the minimizer of $E_{\lambda}$ in $\mathcal{X}$ and $m(\lambda):=\int_{\Omega} u_{\lambda}$ the volume rate. Using the comparison principle from Proposition 4.2 it is not difficult to see that $m(\lambda)$ is an increasing function. Unfortunately, the physical volume rate is given, using the rescaling (1.23), by $m_{0}:=\left(l^{2} \mu_{s} g_{0} \cos \theta\right) m$, which does not allow us to directly study the monotonicity with respect to the angle $\theta(\cos \theta$ is decreasing for $\theta \in[0, \pi / 2)$ and $\lambda(\theta)$ is increasing by (1.24)).

\subsection{Solving equation $(1.5)$}

As we explained in the introduction, we study the first variation of the functional (1.6), i.e.

$$
\frac{\phi \phi^{\prime \prime}}{\left(1+\left|\phi^{\prime}\right|^{2}\right)^{3 / 2}}-\frac{1}{\sqrt{1+\left|\phi^{\prime}\right|^{2}}}+\lambda=0, \quad y \in(-1,1)
$$

Lemma 4.4. (An explicit solution of 4.3)

Let $\lambda>1, K(\lambda)$ be given by $(2.7)$ and $\phi_{K, \lambda}$ defined in (2.6). Then the function $\phi_{K, \lambda} \in C^{\infty}(-1,1) \cap C([-1,1])$ is a non-positive solution of (4.3) and

$$
\lim _{y \rightarrow-1} \phi_{K, \lambda}^{\prime}(y)=-\infty, \quad \lim _{y \rightarrow 1} \phi_{K, \lambda}^{\prime}(y)=+\infty
$$

Moreover the function $\phi_{K, \lambda}$ is convex with minimum $\phi_{K, \lambda}(0)=\frac{K(\lambda)}{\lambda-1}$, maximum $\phi_{K, \lambda}( \pm 1)=\frac{K(\lambda)}{\lambda}$ and if $\bar{\lambda}>\lambda$ then $\phi_{K, \bar{\lambda}}(y)<\phi_{K, \lambda}(y)$, for $y \in[-1,1]$.

Proof of Lemma 4.4

Step 1. The inverse function.

Let $\lambda>1, Z \in\left[\frac{1}{\lambda}, \frac{1}{\lambda-1}\right]$ and $f_{\lambda}(Z)$ be given by (2.5). Notice that $f_{\lambda}$ is smooth in $\left(\frac{1}{\lambda}, \frac{1}{\lambda-1}\right)$ and that it has been chosen so that

$$
f_{\lambda}^{\prime}(Z)=\frac{(\lambda Z-1) \sqrt{\lambda^{2}-1}}{\sqrt{1-\left(\left(\lambda^{2}-1\right) Z-\lambda\right)^{2}}}
$$

We set

$$
A_{\lambda}:=f_{\lambda}\left(\frac{1}{\lambda-1}\right)-f_{\lambda}\left(\frac{1}{\lambda}\right)=\frac{\pi}{2\left(\lambda^{2}-1\right)^{3 / 2}}+\frac{1}{\lambda^{2}-1}\left(1+\frac{\operatorname{Arcsin}\left(\frac{1}{\lambda}\right)}{\sqrt{\lambda^{2}-1}}\right) .
$$


E. NTOVORIS AND M. REGIS

By the monotonicity of $f$ we can define the positive function $\phi_{\lambda}$ implicitly in the intervals $\left[-A_{\lambda}, 0\right]$ and $\left[0, A_{\lambda}\right]$ as follows

$$
f_{\lambda}\left(\phi_{\lambda}(y)\right)=f_{\lambda}\left(\frac{1}{\lambda-1}\right)-|y|, \quad y \in\left[-A_{\lambda}, A_{\lambda}\right]
$$

from which we get that $\phi_{\lambda}$ is an even function thanks to the monotonicity of $f_{\lambda}$. Also, by (4.7) we have $\phi_{\lambda}(0)=1 /(\lambda-1)$ and using (4.5) we can calculate the limit $\lim _{y \rightarrow 0^{+}} \phi_{\lambda}^{\prime}(y)=0$. Since $\phi_{\lambda}$ is even and smooth in the intervals $\left[-A_{\lambda}, 0\right)$ and $\left(0, A_{\lambda}\right]$ we eventually get $\phi_{\lambda}^{\prime}(0)=0$. We have concluded that $\phi_{\lambda} \in C^{1}\left(-A_{\lambda}, A_{\lambda}\right)$.

Relation (4.7) gives also for $y \in\left[-A_{\lambda}, A_{\lambda}\right]$

$$
1 / \lambda=\phi_{\lambda}\left( \pm A_{\lambda}\right) \leq \phi_{\lambda}(y) \leq \phi_{\lambda}(0)=1 /(\lambda-1)
$$

and by $(4.5)$

$$
\phi_{\lambda}^{\prime}\left(-A_{\lambda}\right)=+\infty, \phi_{\lambda}^{\prime}\left(A_{\lambda}\right)=-\infty
$$

Step 2. $\phi_{\lambda}$ satisfies (4.3).

Using (4.5) we can differentiate (4.7) and taking the squares in both sides of the equation, we get after a few simplifications that

$$
\left|\phi_{\lambda}^{\prime}\right|^{2}=\frac{1}{\left(\lambda-\frac{1}{\phi_{\lambda}}\right)^{2}}-1, \quad y \in\left(-A_{\lambda}, A_{\lambda}\right)
$$

Noting that $\phi_{\lambda} \geq 1 / \lambda>0$, the above equation can be rewritten as

$$
\phi_{\lambda}\left(\lambda-\frac{1}{\sqrt{1+\left|\phi_{\lambda}^{\prime}\right|^{2}}}\right)=1
$$

Let $K_{0}<0$, we define

$$
\phi_{K_{0}, \lambda}(y):=K_{0} \phi_{\lambda}\left(\frac{y}{K_{0}}\right), \quad y \in\left[A_{\lambda} K_{0},-A_{\lambda} K_{0}\right] .
$$

By (4.10), the negative function $\phi_{K_{0}, \lambda}$ satisfies

$$
\phi_{K_{0}, \lambda}(y)\left(\lambda-\frac{1}{\sqrt{1+\left|\phi_{K_{0}, \lambda}^{\prime}(y)\right|^{2}}}\right)=K_{0}, \quad y \in\left(A_{\lambda} K_{0},-A_{\lambda} K_{0}\right) .
$$

In particular, if $K(\lambda)$ is given by (2.7), differentiating (4.12) with respect to $y$ we get

$$
\phi_{K, \lambda}^{\prime}\left(\frac{\phi_{K, \lambda} \phi_{K, \lambda}^{\prime \prime}}{\left(1+\left|\phi_{K, \lambda}^{\prime}\right|^{2}\right)^{3 / 2}}-\frac{1}{\sqrt{1+\left|\phi_{K, \lambda}^{\prime}\right|^{2}}}+\lambda\right)=0, \quad y \in(-1,0) \cup(0,1) .
$$


Using equation (4.13) we calculate for $y \in(-1,0) \cup(0,1)$

$$
\phi_{K, \lambda}^{\prime \prime}=\frac{\left(1+\left|\phi_{K, \lambda}^{\prime}\right|^{2}\right)\left(\lambda \sqrt{1+\left|\phi_{K, \lambda}^{\prime}\right|^{2}}-1\right)}{-\phi_{K, \lambda}}>0
$$

where we also used equation (4.12) in order to get the sign of the second derivative. Since $\phi_{K, \lambda} \in C^{1}(-1,1)$ we get from (4.14) that in fact $\phi_{K, \lambda} \in C^{2}((-1,1))$. Differentiating further (4.14) and using (4.8) we get by iteration $\phi_{K, \lambda} \in C^{\infty}(-1,1)$.

Step 3. Extrema.

By (4.8) and (4.11) we have

$$
\left\{\begin{array}{l}
\min _{|y| \leq 1} \phi_{K, \lambda}=\phi_{K, \lambda}(0)=\frac{K(\lambda)}{\lambda-1}=\frac{1}{(\lambda-1)\left(f_{\lambda}\left(\frac{1}{\lambda}\right)-f_{\lambda}\left(\frac{1}{\lambda-1}\right)\right)} \\
\max _{|y| \leq 1} \phi_{K, \lambda}=\phi_{K, \lambda}(-1)=\phi_{K, \lambda}(1)=\frac{K(\lambda)}{\lambda}=\frac{1}{\lambda\left(f_{\lambda}\left(\frac{1}{\lambda}\right)-f_{\lambda}\left(\frac{1}{\lambda-1}\right)\right)} .
\end{array}\right.
$$

We have

$$
\frac{\mathrm{d}}{\mathrm{d} \lambda} \phi_{K, \lambda}(1)=-\frac{4\left(2 \lambda^{2}+1\right) \operatorname{Arcsin}\left(\frac{1}{\lambda}\right) \sqrt{\lambda^{2}-1}+2 \pi\left(2 \lambda^{2}+1\right) \sqrt{\lambda^{2}-1}+4\left(\lambda^{2}-1\right)\left(\lambda^{2}+2\right)}{\lambda^{2}\left(2 \sqrt{\lambda^{2}-1}+\pi+2 \operatorname{Arcsin}\left(\frac{1}{\lambda}\right)\right)^{2}}<0
$$

and

$$
\frac{\mathrm{d}}{\mathrm{d} \lambda} \phi_{K, \lambda}(0)=-\frac{4\left(\lambda-\frac{1}{2}\right)(\lambda-1) \sqrt{\lambda^{2}-1}\left(\pi+2 \operatorname{Arcsin}\left(\frac{1}{\lambda}\right)\right)+4\left(\lambda^{2}-1\right)\left[(\lambda-1)^{2}+\frac{\lambda-1}{\lambda}\right]}{(\lambda-1)^{2}\left(2 \sqrt{\lambda^{2}-1}+\pi+2 \operatorname{Arcsin}\left(\frac{1}{\lambda}\right)\right)^{2}}<0
$$

Figure $3 \mathrm{~b}$ is the graph of the function $\phi_{K, \lambda}(1)$ in terms of the variable $\lambda$.

Step 4. Monotonicity of the graphs in $\lambda$.

Let $\bar{\lambda}>\lambda$ we will show that $\phi_{K, \bar{\lambda}}(y)<\phi_{K, \lambda}(y)$, for $y \in[-1,1]$. Since the functions are even and we already have the monotonicity of the boundary points by Step 3 , we will focus in the interval $(0,1)$. If we use equation (4.13), we get that the function $w(y)=\phi_{K, \bar{\lambda}}(y)-\phi_{K, \lambda}(y)$ satisfies the elliptic equation

$$
-a_{1}(y) w^{\prime \prime}(y)+a_{2}(y) w^{\prime}(y)+a_{3}(y) w(y)=\lambda-\bar{\lambda},
$$

with

$$
a_{1}(y):=\frac{-\phi_{K, \bar{\lambda}}(y)}{\left(1+\left|\phi_{K, \bar{\lambda}}^{\prime}(y)\right|^{2}\right)^{3 / 2}}, \quad a_{3}(y):=\frac{\phi_{K, \lambda}^{\prime \prime}(y)}{\left(1+\left|\phi_{K, \bar{\lambda}}^{\prime}\right|^{2}\right)^{3 / 2}}
$$

and

$$
a_{2}(y):=\int_{0}^{1} G_{1}(p(t, y)) \mathrm{d} t+\phi_{K, \lambda}^{\prime \prime}(y) \phi_{K, \lambda}(y) \int_{0}^{1} G_{2}(p(t, y)) \mathrm{d} t
$$


with $p(t, y):=\phi_{K, \lambda}^{\prime}(y)+t\left(\phi_{K, \bar{\lambda}}^{\prime}(y)-\phi_{K, \lambda}^{\prime}(y)\right), G_{1}(p):=\frac{-p}{\left(1+|p|^{2}\right)^{3 / 2}}$ and $G_{2}(p):=\frac{-3 p}{\left(1+|p|^{2}\right)^{5 / 2}}$. It is $a_{i} \in C(0,1)$, $i=1,2,3$ with $a_{1}, a_{3}>0$ in $(0,1)$ and $w \in C^{2}((0,1)) \cap C([0,1])$ with $w(0), w(1)<0$ by $(4.16)$ and (4.17). We can now conclude that $w<0$ by a maximum principle.

The rescalings of the function $\phi_{K, \lambda}$ which was constructed in Lemma 4.4, will be level sets upon which we will build our three dimensional subsolution and supersolution. For this reason it will be more convenient to parametrize the cone $\mathcal{C}_{\lambda} \cap \Omega$, with $\mathcal{C}_{\lambda}$ as in (2.8), using these rescalings instead of the Cartesian coordinates. Let $L \in(0,+\infty)$, we define

$$
\phi_{L, K, \lambda}(y):=L \phi_{K, \lambda}\left(\frac{y}{L}\right), \quad y \in[-L, L]
$$

The following lemma guarantees a smooth change of coordinate systems, from the Cartesian $(y, z)$ to $(y, L)$.

Lemma 4.5. (A diffeomorphism defining a new set of coordinates).

Let $\phi_{K, \lambda}$ be as in (2.6). Then for $(y, z) \in \overline{\mathcal{C}_{\lambda} \cap \Omega} \backslash\{(0,0)\}$ there is a unique $L=L(y, z) \in(0,+\infty)$ implicitly defined by

$$
z=L \phi_{K, \lambda}\left(\frac{y}{L}\right)
$$

and $L \in C^{\infty}\left(\mathcal{C}_{\lambda} \cap \Omega\right) \cap C\left(\overline{\mathcal{C}_{\lambda} \cap \Omega} \backslash\{(0,0)\}\right)$.

Proof of Lemma 4.5

Since the family of curves $\left\{\left(y, \phi_{L, K, \lambda}(y)\right)\right\}_{L \in(0,+\infty)}$ are obtained as a rescaling of the function $\phi_{K, \lambda}$ we have that the mapping $(y, L) \mapsto(y, z)$ is a surjection; it is also an injection since the family of curves $\left\{\left(y, \phi_{L, K, \lambda}(y)\right)\right\}_{L \in(0,+\infty)}$ do not intersect. On the other hand the same bijective correspondence can be established locally by the implicit function theorem since $\frac{y}{L} \phi_{K, \lambda}^{\prime}\left(\frac{y}{L}\right)-\phi_{K, \lambda}\left(\frac{y}{L}\right)>0$ (since $\phi_{K, \lambda}$ is even and negative), from which we also get the smoothness of $L(y, z)$ in $\mathcal{C}_{\lambda} \cap \Omega$ because $\phi_{K, \lambda}$ is smooth. The continuity of $L$ up to the boundary follows from the definition and the continuity of $\phi_{K, \lambda}$.

Using the diffeomorphism from Lemma 4.5 we can define $q_{\lambda}(y, z) \in C^{\infty}\left(\mathcal{C}_{\lambda} \cap \Omega, \mathbb{R}^{2}\right) \cap C\left(\overline{\mathcal{C}_{\lambda} \cap \Omega} \backslash\{(0,0)\}, \mathbb{R}^{2}\right)$ as follows

$$
q_{\lambda}(y, z):=\frac{\left(-\phi_{L(y, z), K, \lambda}^{\prime}(y), 1\right)}{\sqrt{1+\left|\phi_{L(y, z), K, \lambda}^{\prime}(y)\right|^{2}}}, \quad(y, z) \in \overline{\mathcal{C}_{\lambda} \cap \Omega} \backslash\{(0,0)\}
$$

where $\phi_{L(y, z), K, \lambda}^{\prime}(y)=\phi_{K, \lambda}^{\prime}\left(\frac{y}{L(y, z)}\right)$. Note that the boundary values of $q_{\lambda}$ make sense because of the boundary values of $\phi_{K, \lambda}^{\prime}$ by Lemma 4.4. We have the following lemma.

Lemma 4.6. (The function $q_{\lambda}(y, z)$ solves (1.5)).

Let $\lambda>1, q_{\lambda}$ as in (4.20) then

$$
-\operatorname{div}\left(|z| q_{\lambda}(y, z)\right)=\lambda, \quad \text { for }(y, z) \in\left(\mathcal{C}_{\lambda} \cap \Omega\right) .
$$

Proof of Lemma 4.6

All the equations in this proof hold for $(y, z) \in \mathcal{C}_{\lambda} \cap \Omega$. Having in mind the diffeomorphism $(y, z) \mapsto(\bar{y}, L(y, z))$, with $\bar{y}(y)=y$ from Lemma 4.5 , we can write $q_{\lambda}=q_{\lambda}(\bar{y}, L(y, z))=\left(q_{1}(\bar{y}, L(y, z)), q_{2}(\bar{y}, L(y, z))\right)$. 
In order to simplify the notation we set $\psi:=\phi_{K, \lambda}$. Then using (4.18) we can write $q_{1}=\frac{-\psi^{\prime}(y / L)}{\sqrt{1+\left|\psi^{\prime}(y / L)\right|^{2}}}$ and $q_{2}=\frac{1}{\sqrt{1+\left|\psi^{\prime}(y / L)\right|^{2}}}$, from which we can calculate

$$
\begin{aligned}
\partial_{L} q_{1} & =\frac{\psi^{\prime \prime}\left(\frac{y}{L}\right)\left(\frac{y}{L^{2}}\right)}{\left(1+\left|\psi^{\prime}\left(\frac{y}{L}\right)\right|^{2}\right)^{3 / 2}}, \\
\partial_{L} q_{2} & =\frac{\psi^{\prime \prime}\left(\frac{y}{L}\right) \psi^{\prime}\left(\frac{y}{L}\right)\left(\frac{y}{L^{2}}\right)}{\left(1+\left|\psi^{\prime}\left(\frac{y}{L}\right)\right|^{2}\right)^{3 / 2}} .
\end{aligned}
$$

Differentiating (4.19) in $y$ and $z$ we get

$$
\begin{aligned}
\partial_{z} L & =\frac{-1}{\frac{y}{L} \psi^{\prime}\left(\frac{y}{L}\right)-\psi\left(\frac{y}{L}\right)}, \\
\partial_{y} L & =\frac{\psi^{\prime}\left(\frac{y}{L}\right)}{\frac{y}{L} \psi^{\prime}\left(\frac{y}{L}\right)-\psi\left(\frac{y}{L}\right)} .
\end{aligned}
$$

Using (4.22), (4.23) we get $\partial_{L}\left(q_{1}\right) \partial_{y} L+\partial_{L}\left(q_{2}\right) \partial_{z} L=0$ and hence we get

$$
\operatorname{div}_{(y, z)} q_{\lambda}=\partial_{\bar{y}} q_{1}=\frac{\mathrm{d}}{\mathrm{d} \bar{y}} \frac{-\phi_{L, K, \lambda}^{\prime}(\bar{y})}{\sqrt{1+\left|\phi_{L, K, \lambda}^{\prime}(\bar{y})\right|^{2}}}=\frac{-\phi_{L, K, \lambda}^{\prime \prime}(\bar{y})}{\left(1+\left|\phi_{L, K, \lambda}^{\prime}(\bar{y})\right|^{2}\right)^{3 / 2}} .
$$

Using the fact that $\bar{y}=y, z<0,(4.18),(4.19)$ and (4.24), we get

$$
-\operatorname{div}_{(y, z)}\left(|z| q_{\lambda}\right)=-\frac{\phi_{L, K, \lambda}(y) \phi_{L, K, \lambda}^{\prime \prime}(y)}{\left(1+\left|\phi_{L, K, \lambda}^{\prime}(y)\right|^{2}\right)^{3 / 2}}+\frac{1}{\sqrt{1+\left|\phi_{L, K, \lambda}^{\prime}(y)\right|^{2}}},
$$

and finally using the above equation together with (4.13) and the definition of $\phi_{L, K, \lambda}$ we conclude

$$
-\operatorname{div}_{(y, z)}\left(|z| q_{\lambda}\right)=\lambda, \quad(y, z) \in \mathcal{C}_{\lambda} \cap \Omega .
$$

Note also that by (4.23) and the boundary conditions of $\phi_{K, \lambda}^{\prime}$ we can extend $L \in C^{1}\left(\overline{\mathcal{C}_{\lambda} \cap \Omega} \backslash\{(0,0)\}\right)$.

Lemma 4.7. (Bound on the Laplacian $\Delta L$ )

Let $L$ be as in (4.19). Then there are positive constants $C_{1}, C_{2}$ such that for

$$
C(\lambda):=1+\left(\frac{\lambda-1}{K(\lambda)}\right)^{2}
$$

we have

$$
\begin{aligned}
\left(\partial_{y} L(y, z)\right)^{2}+\left(\partial_{z} L(y, z)\right)^{2} \leq C(\lambda) & \text { for }(y, z) \in \mathcal{C}_{\lambda} \cap \Omega, \\
L(y, z) \Delta_{(y, z)} L(y, z) \leq C_{1}+C_{2} & \text { for }(y, z) \in \mathcal{C}_{\lambda} \cap \Omega,
\end{aligned}
$$

in particular we have

$$
0 \leq \Delta_{(y, z)}(L(y, z))^{2} \leq 2\left(C(\lambda)+C_{1}+C_{2}\right), \quad(y, z) \in \mathcal{C}_{\lambda} \cap \Omega .
$$


Proof of Lemma 4.7

As in the proof of Lemma 4.6 we simplify the notation by setting $\psi:=\phi_{K, \lambda}$.

Step 1. Bound on $\partial_{z} L$ and $\partial_{y} L$.

Let $(y, z) \in \mathcal{C}_{\lambda} \cap \Omega$, by the diffeomorphism in Lemma 4.5 we have $\frac{|y|}{L(y, z)} \leq 1$. By (4.14) we have $\psi^{\prime \prime}>0$ in $(-1,1)$, then, using also (4.15) and formula (4.23) we can estimate $\left|\partial_{z} L(y, z)\right| \leq \frac{\lambda-1}{-K(\lambda)}$.

Similarly for $\partial_{y} L$ given by (4.23) we have $\left|\partial_{y} L(y, z)\right| \leq 1$ for $(y, z) \in \mathcal{C}_{\lambda} \cap \Omega$, since the function $\psi^{\prime}(y) /\left(y \psi^{\prime}(y)-\psi(y)\right)$ is strictly increasing in $(-1,1)$ and $\lim _{y \rightarrow 1} \frac{\psi^{\prime}(y)}{y \psi^{\prime}(y)-\psi(y)}=1$. Combining the bounds of $\partial_{z} L$ and $\partial_{y} L$ we get (4.26).

Step 2. Bound on second derivatives.

If we differentiate (4.19) twice in $z$ and $y$ respectively and use (4.23) we get

$$
L \partial_{z z}^{2} L=\frac{\psi^{\prime \prime}\left(\frac{y}{L}\right)\left(\frac{y}{L}\right)^{2}}{\left(\frac{y}{L} \psi^{\prime}\left(\frac{y}{L}\right)-\psi\left(\frac{y}{L}\right)\right)^{3}}
$$

and

$$
L \partial_{y y}^{2} L=\frac{\psi^{\prime \prime}\left(\frac{y}{L}\right) \psi^{2}\left(\frac{y}{L}\right)}{\left(\frac{y}{L} \psi^{\prime}\left(\frac{y}{L}\right)-\psi\left(\frac{y}{L}\right)\right)^{3}} .
$$

We estimate in $\mathcal{C}_{\lambda} \cap \Omega$

$$
L \partial_{z z}^{2} L \leq \max \left\{\max _{\frac{|y|}{L} \leq \frac{1}{2}} L \partial_{z z}^{2} L, \sup _{\frac{1}{2}<\frac{|y|}{L}<1} L \partial_{z z}^{2} L\right\} .
$$

Using the fact that $y \psi^{\prime}(y) \geq 0$ and the maximum of $\psi$ by (4.15) we estimate

$$
\max _{\frac{|y|}{L} \leq \frac{1}{2}} L \partial_{z z}^{2} L \leq \frac{1}{4}\left(\frac{\lambda}{-K(\lambda)}\right)^{3} \max _{|y| \leq \frac{1}{2}} \psi^{\prime \prime}(y) .
$$

For $1 / 2<|y / L|<1$, it is $\psi^{\prime} \neq 0$ and we can rewrite (4.29) as

$$
L \partial_{z z}^{2} L=\frac{\psi^{\prime \prime}\left(\frac{y}{L}\right)}{\left|\psi^{\prime}\left(\frac{y}{L}\right)\right|^{3}} \cdot \frac{\left(\frac{y}{L}\right)^{2}}{\left(\left|\frac{y}{L}\right|+\frac{-\psi\left(\frac{y}{L}\right)}{\left|\psi^{\prime}\left(\frac{y}{L}\right)\right|}\right)^{3}} .
$$

By equation (4.14) we calculate in $(1 / 2,1)$ the term $\psi^{\prime \prime} /\left|\psi^{\prime}\right|^{3}$ and using properties of $\psi$ and the monotonicity of $\psi^{\prime}$ we eventually get the bound

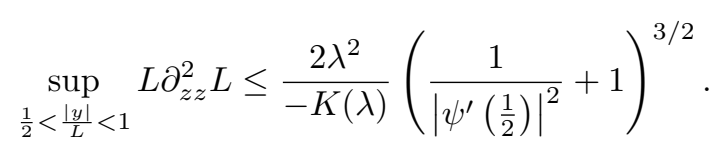


Finally, by (4.33) and (4.31) we get $\sup _{\mathcal{L}} L \partial_{z z}^{2} L \leq C_{1}$, with $C_{1}$ a positive constant. Similarly, one can show that $\sup _{\mathcal{C}} L \partial_{y y}^{2} L \leq C_{2}$ with $\mathcal{C}_{\lambda} \cap \Omega$

$$
C_{2}:=\max \left\{\frac{\lambda}{-K(\lambda)} \max _{|y| \leq \frac{1}{2}} \psi^{\prime \prime}(y), 8\left(\frac{\lambda}{\lambda-1}\right)^{2}(-K(\lambda))\left(\frac{1}{\left|\psi^{\prime}\left(\frac{1}{2}\right)\right|^{2}}+1\right)^{3 / 2}\right\}
$$

\subsection{A subsolution}

Remark 4.8. Let $\sigma: \Omega_{1} \cup \Omega_{2} \rightarrow \mathbb{R}^{2}$, with $\Omega_{1}, \Omega_{2} \subset \mathbb{R}^{2}$, two bounded domains with Lipschitz boundary and a common smooth boundary $\partial \Omega$, with surface measure $\mathrm{d} S$. Suppose that $\sigma \in \bigcap_{i=1}^{2}\left(C^{1}\left(\Omega_{i}, \mathbb{R}^{2}\right) \cap C\left(\overline{\Omega_{i}}, \mathbb{R}^{2}\right)\right)$, $\operatorname{div} \sigma \in L^{2}\left(\Omega_{1}\right) \cap L^{2}\left(\Omega_{2}\right)$. We denote by $\left.\operatorname{Tr}\right|_{\Omega_{i}} \sigma, i=1,2$, the limit value of $\sigma$ from the sides $\Omega_{i}$ respectively. Then for $\varphi \in W_{0}^{1,2}\left(\Omega_{1} \cup \Omega_{2}\right)$ with $\operatorname{supp}(\varphi) \cap \partial \Omega \neq \emptyset$, it is

$$
\int_{\Omega_{1} \cup \Omega_{2}} \sigma \cdot \nabla \varphi=-\int_{\Omega_{1} \cup \Omega_{2}} \operatorname{div}(\sigma) \varphi+\int_{\partial \Omega} n \cdot\left(\left.\operatorname{Tr}\right|_{\Omega_{1}} \sigma-\left.\operatorname{Tr}\right|_{\Omega_{2}} \sigma\right) \varphi \mathrm{d} S
$$

where $n$ is the normal to $\partial \Omega$ pointing at the direction of $\Omega_{2}$. We will also call the second integral in the right hand side of (4.34) the concentration of $\operatorname{div}(\sigma)$ on $\partial \Omega$.

We can now construct a subsolution. We will first give the main idea of the construction. The vector field $q_{\lambda}$ defined in (4.20) solves (1.5) in $\mathcal{C}_{\lambda}$ and is the normal to the family of rescaled curves (4.18), which will be level sets of the subsolution. All that remains to do in $\mathcal{C}_{\lambda}$ is to assign a value to each level set, in such a way that the sign and absolute value of the Laplacian of the subsolution can be controlled. To do that, we choose the subsolution to be a small enough inverse parabola in the set $\Omega \backslash \mathcal{C}_{\lambda}$ (Eq. (4.35)). There is now only one way to assign a value on the level sets inside the cone $\mathcal{C}_{\lambda}$ so that the subsolution is continuous, see Figure 4. The choice of the inverse parabola has also served on creating a concave function, thus fixing the sign of the Laplacian. One can now choose the value of the constructed function (Eq. (4.35), in fact the parameter $\zeta$ ) to be small enough so that the subsolution inequalities are satisfied.

In what follows we will favor intuition over mathematical elegance, as far as the notation is concerned, and we will instead denote the set $\mathrm{Epi}^{\smile}(\lambda)$ defined in (2.9), simply by $\left\{z>\phi_{K, \lambda}\right\}$. Let $\zeta>0$, using the diffeomorphism from Lemma 4.5 we can define the continuous function (see Fig. 4)

$$
u_{\zeta, \lambda}(y, z):= \begin{cases}-\zeta y^{2}+\zeta & \text { in } \Omega \backslash \mathcal{C}_{\lambda} \\ -\zeta L^{2}(y, z)+\zeta & \text { in } \mathcal{C}_{\lambda} \cap\left\{z \geq \phi_{K, \lambda}\right\} \\ 0 & \text { in }\left\{z<\phi_{K, \lambda}\right\}\end{cases}
$$

and for $q_{\lambda}$ as in (4.20) we define

$$
d_{\lambda}^{\operatorname{ext}}(y, z):= \begin{cases}\left(-\frac{y}{|y|}, 0\right) & \text { in } \Omega \backslash \mathcal{C}_{\lambda} \\ q_{\lambda}(y, z) & \text { in } \mathcal{C}_{\lambda} \cap \Omega\end{cases}
$$

Then we have that $u_{\zeta, \lambda} \in \mathcal{X}$ with $\partial_{z} u_{\zeta, \lambda}(y, 0)=0$ for $y \in(-1,1)$ and $d_{\lambda}^{\text {ext }} \in \Lambda$. In the set $\mathcal{C}_{\lambda} \cap\left\{z>\phi_{K, \lambda}\right\}$ we have $\nabla u_{\zeta, \lambda}=-2 \zeta L\left(\partial_{y} L, \partial_{z} L\right)$. Then using also (4.23), (4.35), (4.36), definition (4.20) and the properties of $\phi_{K, \lambda}$ from Lemma 4.4 we have that $d_{\lambda}^{\text {ext }} \cdot \nabla u_{\zeta, \lambda}=\left|\nabla u_{\zeta, \lambda}\right|$ a.e. in $\Omega$. 


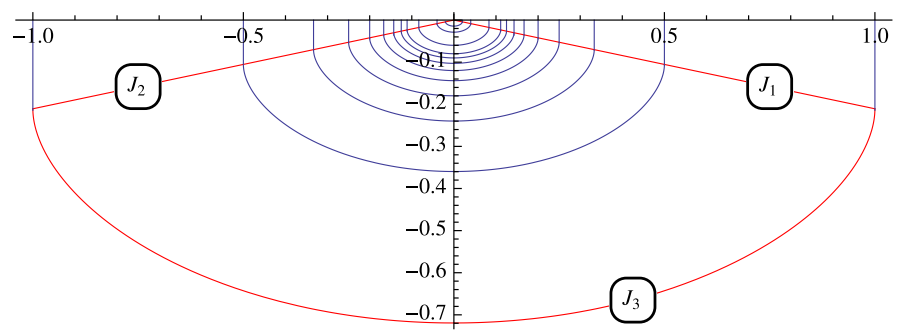

(a) Curves with normal $d_{\lambda}^{\text {ext }}$

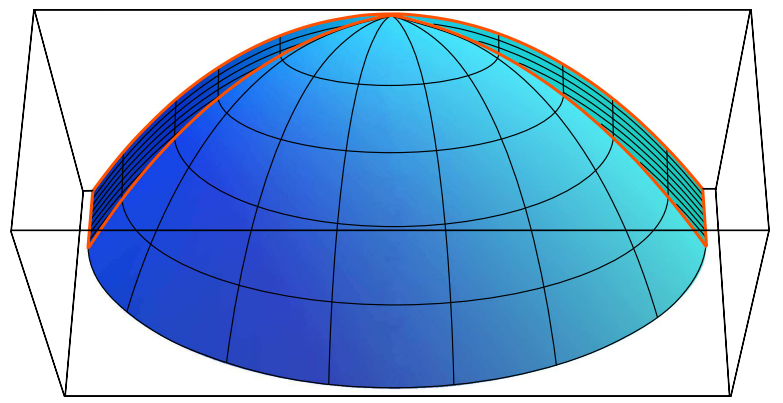

(b) $u_{\zeta, \lambda}$

Figure 4. Subsolution.

Proposition 4.9. (Subsolution) Let $\lambda>1$. Then for all $\lambda_{0} \in(1, \lambda)$ and $\zeta_{0} \in\left(0, \frac{\lambda-\lambda_{0}}{2\left(C\left(\lambda_{0}\right)+C_{1}+C_{2}\right)}\right)$, with $C\left(\lambda_{0}\right), C_{1}, C_{2}$ given by Lemma 4.7 , the pair $\left(u_{\zeta_{0}, \lambda_{0}}, d_{\lambda_{0}}^{\text {ext }}\right)$ given by (4.35)-(4.36), is a subsolution of the equation (2.3).

Proof of Proposition 4.9

Step 1. The subsolution inequalities.

We will first show the subsolution inequalities in the set

$$
\Omega_{1} \cup \Omega_{2} \cup \Omega_{3}:=\left(\Omega \backslash \overline{\mathcal{C}_{\lambda_{0}}}\right) \cup\left(\mathcal{C}_{\lambda_{0}} \cap\left\{z>\phi_{K, \lambda_{0}}\right\}\right) \cup\left(\mathcal{C}_{\lambda_{0}} \backslash\left\{z \geq \phi_{K\left(\lambda_{0}\right)}\right\}\right)
$$

where the functions $u_{\zeta_{0}, \lambda_{0}}, d_{\lambda_{0}}^{\text {ext }}$ are smooth. Using (4.21) and (4.36) we calculate

$$
-\operatorname{div}\left(|z| d_{\lambda_{0}}^{\text {ext }}(y, z)\right)= \begin{cases}0 & \text { in } \Omega \backslash \overline{\mathcal{C}_{\lambda_{0}}} \\ \lambda_{0} & \text { in } \mathcal{C}_{\lambda_{0}} \cap \Omega\end{cases}
$$

Also

$$
-\Delta u_{\zeta_{0}, \lambda_{0}}= \begin{cases}2 \zeta_{0} & \text { in } \Omega \backslash \overline{\mathcal{C}_{\lambda_{0}}}, \\ 0 & \text { in } \Omega \cap\left\{z<\phi_{K, \lambda_{0}}\right\} .\end{cases}
$$

and using Lemma 4.7 we get in $\mathcal{C}_{\lambda_{0}} \cap\left\{z>\phi_{K, \lambda_{0}}\right\}$

$$
-\Delta u_{\zeta_{0}, \lambda_{0}}=\zeta_{0} \Delta L^{2} \leq 2 \zeta_{0}\left(C\left(\lambda_{0}\right)+C_{1}+C_{2}\right)
$$

If we now combine (4.37)-(4.39), use the fact that the positive constant $2\left(C\left(\lambda_{0}\right)+C_{1}+C_{2}\right)$ depends only on $\lambda_{0}$, we can choose $\zeta_{0} \leq \frac{\lambda-\lambda_{0}}{2\left(C\left(\lambda_{0}\right)+C_{2}+C_{2}\right)}\left(<\lambda / 2\right.$ since $C\left(\lambda_{0}\right)+C_{1}+C_{2}>1$ by $\left.(4.25)\right)$ and get

$$
-\Delta u_{\zeta_{0}, \lambda_{0}}-\operatorname{div}\left(|z| d_{\lambda_{0}}^{\text {ext }}\right) \leq \lambda \text { in } \Omega_{1} \cup \Omega_{2} \cup \Omega_{3} .
$$

It remains to show that inequality (4.40) holds in the rest of $\Omega$. We will use Remark 4.8 for $\sigma:=\nabla u_{\zeta_{0}, \lambda_{0}}+|z| d_{\lambda_{0}}^{\text {ext }}$. Note that $\sigma$ is not defined at $(0,0)$ but we still have that it is bounded near $z=0$ by Lemma 4.7 .

Step 2. Concentration on curves.

Note that since $\partial_{z} u_{\zeta_{0}, \lambda_{0}}(y, 0)=0$ and therefore $\sigma(y, 0)=0$, for $y \in(-1,1) \backslash\{(0,0)\}$ in view of (4.34), we do not need to take into account the boundary $\{z=0\}$. We denote by $J:=J_{1} \cup J_{2} \cup J_{3}$ the three parts of the 
boundary of $\Omega_{1} \cup \Omega_{2} \cup \Omega_{3}$ as in Figure 4a. We will show the subsolution inequalities on $J$. We need to estimate for $(i, j) \in\{(1,2),(2,3)\}$, the terms

$$
n_{j} \cdot\left(\left.\operatorname{Tr}\right|_{\Omega_{i}} \sigma-\left.\operatorname{Tr}\right|_{\Omega_{j}} \sigma\right)
$$

where $n_{j}$ is the normal of the common boundary pointing in the direction of $\Omega_{j}$. For $J_{1}$, the right common boundary of $\Omega_{1}$ and $\Omega_{2}$ we have $n_{1}=\left(\frac{K\left(\lambda_{0}\right)}{\lambda_{0}},-1\right)$. Using (4.4) and (4.20) one can see that that $d_{\lambda}^{\text {ext }}$ is continuous in $\Omega$. Then using (4.35) we get

$$
\left.\operatorname{Tr}\right|_{\Omega_{1}} \sigma-\left.\operatorname{Tr}\right|_{\Omega_{2}} \sigma=\left(-2 \zeta_{0} y, 0\right)+2 \zeta_{0} L\left(\partial_{y} L, \partial_{z} L\right)=0, \quad(y, z) \in J_{1},
$$

where we used the fact that $y=L$ on $J_{1}$ and $\left(\partial_{y} L, \partial_{z} L\right)=(1,0)$ by the Neumann conditions in (4.4). In a similar way we can write $(4.41)$ on $J_{2}$ as

$$
-n_{2} \cdot\left(\left.\operatorname{Tr}\right|_{\Omega_{1}} \sigma-\left.\operatorname{Tr}\right|_{\Omega_{2}} \sigma\right)=0
$$

where $n_{2}=\left(\frac{-K\left(\lambda_{0}\right)}{\lambda_{0}},-1\right)$. On $J_{3}$ we simplify the notation and set $\psi=\phi_{K, \lambda_{0}}$. Then (4.41) becomes

$$
\begin{aligned}
n_{3} \cdot\left(\left.\operatorname{Tr}\right|_{\Omega_{2}} \sigma-\left.\operatorname{Tr}\right|_{\Omega_{3}} \sigma\right) & =\left(\frac{\psi^{\prime}}{\sqrt{1+\left|\psi^{\prime}\right|^{2}}}, \frac{-1}{\sqrt{1+\left|\psi^{\prime}\right|^{2}}}\right) \cdot\left(-2 \zeta_{0} L\left(\partial_{y} L, \partial_{z} L\right)\right) \\
& =-2 \zeta_{0} \frac{\sqrt{1+\left|\psi^{\prime}\right|^{2}}}{y \psi^{\prime}-\psi} \leq 0
\end{aligned}
$$

We can now conclude from estimates (4.42)-(4.44).

Proof of Theorem 2.3 (lower bound)

If we compare the subsolution $u_{\zeta_{0}, \lambda_{0}}$ by Proposition 4.9 with the solution $u_{\lambda}$ of (2.3) using Proposition 4.2, we get $0 \leq u_{\zeta_{0}, \lambda_{0}} \leq u_{\lambda}$ in $\Omega$ for all $\lambda_{0} \in(1, \lambda)$. Then by definitions (4.35) and (2.9) we get

$$
\left\{u_{\zeta_{0}, \lambda_{0}}>0\right\}=\left\{z>\phi_{K, \lambda_{0}}\right\}=\operatorname{Epi}^{\smile}\left(\lambda_{0}\right) \subset\left\{u_{\lambda}>0\right\}, \quad \text { for all } \lambda_{0} \in(1, \lambda) .
$$

We set $\psi(y, \lambda):=\phi_{K, \lambda}(y)$ for $(y, \lambda) \in[0,1] \times(1,+\infty)$. By definition (2.6) we have that $\psi$ satisfies the equation $F(y, \lambda, \psi(y, \lambda))=0$ with

$$
F:\left\{(y, \lambda, z): y \in(0,1), \lambda \in(1,+\infty), z \in\left(\frac{K(\lambda)}{\lambda-1}, \frac{K(\lambda)}{\lambda}\right)\right\} \rightarrow \mathbb{R}
$$

given by

$$
F(y, \lambda, z):=K(\lambda) f_{\lambda}\left(\frac{z}{K(\lambda)}\right)-K(\lambda) f_{\lambda}\left(\frac{1}{\lambda-1}\right)-y
$$

Using the formulas (2.5), (2.7) and (4.6) one can check that $F$ is smooth in the domain of its definition. Since $f_{\lambda}^{\prime}\left(\frac{\psi(y, \lambda)}{K(\lambda)}\right)>0$ for $(y, \lambda) \in(0,1) \times(1,+\infty)$, we have by the implicit function theorem that $\psi \in C^{\infty}((0,1) \times$ $(1,+\infty))$. Since $\phi_{K, \lambda}$ is even, we get that for fixed $y \in(-1,0) \cup(0,1)$ the function $\phi_{K, \lambda}(y)$ is continuous in $\lambda$ in $(1,+\infty)$. By the formulas of $\phi_{K, \lambda}( \pm 1), \phi_{K, \lambda}(0)$ by Lemma 4.4 and the continuity of the function $K(\lambda)$ we get that $\lim _{\lambda_{0} \uparrow \lambda} \phi_{K, \lambda_{0}}(y)=\phi_{K, \lambda}(y)$ for all $y \in[-1,1]$. We can now pass to the limit in (4.45) and conclude. 


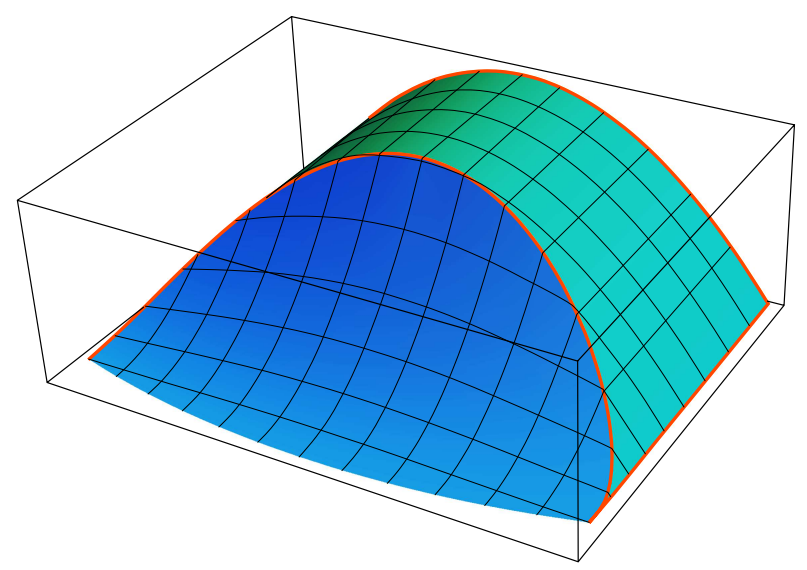

FIGURE 5. $U^{\lambda_{1}, \vartheta}$.

\subsection{A supersolution}

The idea behind the construction of the supersolution is similar to that of the subsolution. Near $z=0$ we take an inverse parabola (Eq. (4.46)) which doesn't have to be very small this time. We assign to the level sets, i.e. the rescaled functions in (4.18) the values of an increasing quadratic function (Eq. (4.47)), using the diffeomorphism from Lemma 4.5. This time, one has to be careful to rescale further the functions in (4.18) $(L>1)$, so that enough space is given to the quadratic function, in order for it to increase enough and intersect the parabola in the domain $\Omega$. This particular rescaling is also necessary so that the Laplacian of the resulting solution will have the correct sign. The final supersolution will be the minimum of the two supersolutions and the concentration of the second derivatives on the intersection mentioned above will have the correct sign.

Let $\lambda>1, \lambda_{1}>\lambda$ and $\vartheta, b, \Pi$ given by (2.10), (2.11), (2.12) respectively. Using the diffeomorphism from Lemma 4.5 with $\phi_{K, \lambda_{1}}$ in (4.19), we can consider sets of the form $\left\{(y, z) \in \mathcal{C}_{\lambda} \cap \Omega: 1 \leq L(y, z) \leq b\right\}$, where the level set $\left\{(y, z) \in \mathcal{C}_{\lambda} \cap \Omega: L(y, z)=1\right\}$ is the graph $\left\{z=\phi_{K, \lambda_{1}}\right\}$; we will simply denote by $\{1 \leq L(y, z) \leq b\}$ these sets. We define

$$
\begin{gathered}
u_{1}^{\lambda_{1}}(y, z):=\frac{\lambda_{1}}{2}\left(1-y^{2}\right), \quad(y, z) \in \Omega, \\
u_{2}^{\lambda_{1}, \vartheta}(y, z):= \begin{cases}+\infty & \text { in }\left\{z>\phi_{K, \lambda_{1}}\right\}, \\
\vartheta(L(y, z)-b)^{2} & \text { in }\{1 \leq L(y, z) \leq b\}, \\
0 & \text { in }\{b \leq L(y, z)\},\end{cases}
\end{gathered}
$$

where we simply write $\vartheta$ for $\vartheta_{\lambda, \lambda_{1}}$. Also, we define

$$
U^{\lambda_{1}, \vartheta}:=\min \left\{u_{1}^{\lambda_{1}}, u_{2}^{\lambda_{1}, \vartheta}\right\}, \quad \text { in } \Omega
$$

We note that the intersection of the graphs of the functions $u_{2}^{\lambda_{1}, \vartheta}$ and $u_{1}^{\lambda_{1}}$ lies in the domain $\Omega \cap\{L(y, z)<b\}$ and is given by the equation

$$
\vartheta(L(y, z)-b)^{2}=\frac{\lambda_{1}}{2}\left(1-y^{2}\right), \quad(y, z) \in \Omega \cap\{L(y, z)<b\},
$$


and since $L<b$

$$
L(y, z)=b-\sqrt{\frac{\lambda_{1}}{2 \vartheta}\left(1-y^{2}\right)} \geq b-\sqrt{\frac{\lambda_{1}}{2 \vartheta}}=1,
$$

by the definition of $b$. Also, since $\partial_{z} L<0$ in $\mathcal{C}_{\lambda} \cap \Omega$ the curve defined by the contour (4.49) is the graph of a function which lies in fact in the set $\{1 \leq L(y, z) \leq b\}$, and therefore, the function $U^{\lambda_{1}, \vartheta}$ is continuous, see Figure 5. For $q_{\lambda_{1}}$ as in (4.20) we define for a.e. $y \in \Omega$ the vector field

$$
q_{\lambda_{1}}^{\text {ext }}(y, z):= \begin{cases}\left(-\frac{y}{|y|}, 0\right) & \text { in }\left(\left\{1 \leq L(y, z)<b-\sqrt{\frac{\lambda_{1}}{2 \vartheta}\left(1-y^{2}\right)}\right\} \cup\left\{z>\phi_{K, \lambda_{1}}\right\}\right) \cap\{y \neq 0\} \\ q_{\lambda_{1}}(y, z) \quad \text { in }\left\{b-\sqrt{\frac{\lambda_{1}}{2 \vartheta}\left(1-y^{2}\right)}<L(y, z)\right\} .\end{cases}
$$

We have the following proposition.

Proposition 4.10. (Supersolution)

Let $\lambda>1$. Then the function $U^{\lambda_{1}, \vartheta}$ defined in (4.48) is a supersolution of (2.3).

Proof of Proposition 4.10

A straightforward calculation shows that $\nabla U^{\lambda_{1}, \vartheta} \cdot q_{\lambda_{1}}^{\text {ext }}=\left|\nabla U^{\lambda_{1}, \vartheta}\right|$, a.e. in $\Omega$. We also have $\partial_{z} U^{\lambda_{1}, \vartheta}(y, 0)=$ $\partial_{z} u_{1}^{\lambda_{1}}(y, 0)=0$.

Step 1. Supersolution inequalities.

It is

$$
-\Delta U^{\lambda_{1}, \vartheta}= \begin{cases}\lambda_{1} & \text { in }\left\{1 \leq L(y, z)<b-\sqrt{\frac{\lambda_{1}}{2 \vartheta}\left(1-y^{2}\right)}\right\} \cup\left\{z>\phi_{K, \lambda_{1}}\right\} \\ -2 \vartheta\left(\left(\partial_{y} L\right)^{2}+\left(\partial_{z} L\right)^{2}\right) & \\ +2 \vartheta(b-L)\left(\partial_{y y}^{2} L+\partial_{z z}^{2} L\right) & \text { in }\left\{b-\sqrt{\frac{\lambda_{1}}{2 \vartheta}\left(1-y^{2}\right)}<L(y, z)<b\right\} \\ 0 & \text { in }\{b<L(y, z)\},\end{cases}
$$

and as in (4.37) we have

$$
-\operatorname{div}\left(|z| q_{\lambda_{1}}^{\mathrm{ext}}\right)= \begin{cases}0 & \text { in }\left(\left\{1 \leq L(y, z)<b-\sqrt{\frac{\lambda_{1}}{2 \vartheta}\left(1-y^{2}\right)}\right\} \cup\left\{z>\phi_{K, \lambda_{1}}\right\}\right) \cap\{y \neq 0\} \\ \lambda_{1} \quad & \text { in }\left\{b-\sqrt{\frac{\lambda_{1}}{2 \vartheta}\left(1-y^{2}\right)}<L(y, z)\right\} .\end{cases}
$$

Therefore, if $C(\lambda)$ is as in $(4.25)$ we have $\vartheta=\frac{\lambda_{1}-\lambda}{2 C(\lambda)}$ and

$$
-\Delta U^{\lambda_{1}, \vartheta}-\operatorname{div}\left(|z| q_{\lambda_{1}}^{\text {ext }}\right) \geq \lambda, \quad \text { in } \Omega \backslash\left(\left\{L(y, z)=b-\sqrt{\frac{\lambda_{1}}{2 \vartheta}\left(1-y^{2}\right)}\right\} \cup\{0\} \times\left(\frac{K\left(\lambda_{1}\right)}{\lambda_{1}-1}, 0\right)\right) .
$$




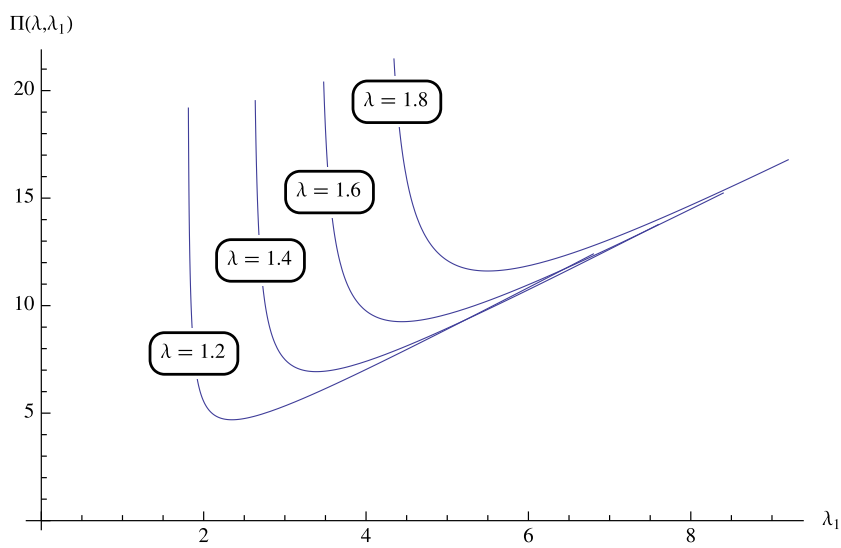

(a) $\Pi\left(\lambda, \lambda_{1}\right)$ for $\lambda=1.2,1.4,1.6,1.8$

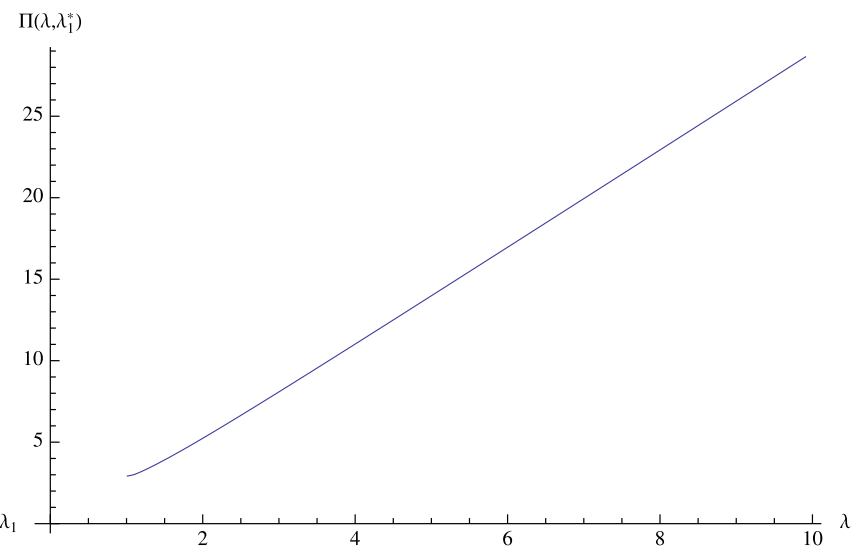

(b) $\Pi\left(\lambda, \lambda_{1}^{\star}\right)$

FiguRE 6. Distance of supports.

Note that the solution of the equation $L(0, z)=1$ is $z=\frac{K\left(\lambda_{1}\right)}{\lambda_{1}-1}$. We also note that by (4.29), (4.30) and Step 2 of the proof of Lemma 4.7 we have that $\Delta U^{\theta, \lambda_{1}}$ is bounded.

Step 2. Concentration on curves.

The discontinuities of the vector fields $\nabla U_{\lambda_{1}, \vartheta}$ and $q_{\lambda_{1}}^{\text {ext }}$ lie on the intersection given by the contour (4.49) and on $\{0\} \times\left(\frac{K\left(\lambda_{1}\right)}{\lambda_{1}-1}, 0\right)$. For the second set only the vector field $q_{\lambda_{1}}^{\text {ext }}$ is discontinuous and the concentration which it creates is

$$
|z|(1,0) \cdot((1,0)-(-1,0)) \geq 0 .
$$

For the intersection, equation (4.49), we suppress the indices $\lambda_{1}, \vartheta$ and we write the concentration as

$$
n \cdot\left[\left(\nabla u_{1}-\nabla u_{2}\right)+|z|\left(\frac{\nabla u_{1}}{\left|\nabla u_{1}\right|}-\frac{\nabla u_{2}}{\left|\nabla u_{2}\right|}\right)\right],
$$

where $n$ is the normal to the intersection pointing at the direction of $\left\{L(y, z)>b-\sqrt{\frac{\lambda_{1}}{2 \vartheta}\left(1-y^{2}\right)}\right\}$. Then the $z$-component of $n$ is negative, and since $L_{z}<0$ by (4.23) we have

$$
n=\frac{\nabla u_{1}-\nabla u_{2}}{\left|\nabla u_{1}-\nabla u_{2}\right|}
$$

Clearly, we have $n \cdot\left(\nabla u_{1}-\nabla u_{2}\right) \geq 0$. The second term of (4.51) is

$$
\frac{|z|}{\left|\nabla u_{1}-\nabla u_{2}\right|}\left(\left|\nabla u_{1}\right|+\left|\nabla u_{2}\right|-\nabla u_{1} \cdot \nabla u_{2} \frac{\left|\nabla u_{1}\right|+\left|\nabla u_{2}\right|}{\left|\nabla u_{1}\right|\left|\nabla u_{2}\right|}\right) \geq 0
$$

by the Cauchy-Schwartz inequality. This concludes the proof.

Proof of Theorem 2.3 (upper bound)

We will estimate supp $u$ above. By Propositions 4.10 and 4.2 we get $0 \leq u_{\lambda} \leq U_{\lambda_{1}, \vartheta}$ in $\Omega$ and since $\operatorname{supp} U_{\lambda_{1}, \vartheta}=$ Epi`$\left(\lambda_{1}\right)$ we get the desired estimate. 
Let $\lambda_{1}^{\star}=\lambda_{1}^{\star}(\lambda)>\lambda$ be a minimizer of $\Pi(\lambda, \cdot)$ - see discussion before Theorem 2.3. In Figure 6a we give the graph of $\Pi\left(\lambda, \lambda_{1}\right)$ for different values of $\lambda$ and in Figure $6 \mathrm{~b}$ we see that the optimal value $\Pi\left(\lambda, \lambda_{1}^{\star}\right)$ increases as we increase the angle $\theta$, or similarly, as we increase $\lambda$, at least for a small range of values.

\section{CONCLUSiOn}

We have studied the minimizer of the non-smooth functional $E_{\lambda}$ in an unbounded domain and have derived its generalized Euler-Lagrange equation in the form of a singular equation. The regularity of the solution of this equation is another interesting problem. Regularity properties for the two-dimensional Bingham problem have been proved in [23] (see also [8]). In this paper, we have proved Hölder continuity but we expect more regularity at least away from the boundary $z=0$. Once higher regularity is proved we can also use Steiner symmetrization techniques to show monotonicity and symmetry properties of the solution.

We have also studied the role of the values of $\lambda$ in the existence of a non-trivial solution. Physically, this is a natural result since the inclination of the plane upon which a non-Newtonian fluid rests, needs to be large enough for the fluid to start moving due to the gravity. In general, identifying the transition from a static to a flowing regime of a material is important for the applications; for example, the start of an avalanche of a granular material. In [16], the author has identified the critical value of $\lambda$ (which appears in the energy for the Bingham case) with the Cheeger constant of the bounded domain of integration, when this domain has a $C^{1}$-boundary. In [14], Theorem 3.1 (ii), - see also [17], Theorem 3.2 - the authors have shown that the Cheeger constant of infinite strips of width $2 a$ is $1 / a$. We note that because of the Neumann condition on $z=0$, our problem can be studied on the whole strip $(-1,1) \times \mathbb{R}$ by a reflection argument. But in our case, the critical value $\lambda=1$ remains the same for the different widths of a wall, and only when the width is 2 this critical value coincides with the Cheeger constant of the strip.

We have given a notion of subsolutions and supersolutions for our problem in terms of a pair of a function and a vector field, which is in general normal to the level sets of the function. The vector field is needed because several vector fields can be chosen when the gradient of the function is 0 . We have proved a comparison principle, and with it we are able to construct an explicit subsolution and a supersolution with compact support. We do this by parametrizing a subset of the strip using curves whose normal solves a curvature like equation. Then we deduce that the solution of our problem will have compact support, the boundary of which is the free boundary and its existence is natural since the equation (1.1) has the equivalent formulation of a variational inequality. The two supports of the subsolution and supersolution respectively give an estimate for the free boundary, and their monotonicity in $\lambda$ shows that the fluid region increases when we increase the inclination angle. We have optimized the estimate of the free boundary by minimizing the function $\Pi(\lambda, \cdot)$ in $(2.12)$. The simulation in Figure $6 \mathrm{~b}$ shows that the gap between the boundaries of the subsolution and the supersolution widens as we increase $\lambda$, at least for small values of $\lambda$.

Another interesting and open problem is the regularity of the free boundary but also of the level sets of the solutions of singular equations like (1.1). An interesting property of these solutions is that they have levels sets with positive measure. In [16], the author has studied properties of the set where the solution of his problem achieves its maximum value. He shows that this set has positive measure and compared it with the Cheeger set. For our problem it is still not clear whether or not our solution has other level sets with positive measure apart from the zero level set.

One particularity of our problem which is studied in an unbounded domain, is the fact that we impose a certain angle $\theta$ and the volume rate $\int_{\Omega} u$ follows as an increasing function of the angle. The unboundedness of the domain allows also the support of the solution to increase boundlessly. The solution coincides with the solution of the similar problem for which the distance between the bottom plane and the atmosphere is finite, with zero boundary conditions at the bottom, as long as this distance is big enough. In fact this solution in the bounded domain, does not depend on the depth of the bottom plain, as long as the plane lies outside the support of the initial solution which was considered in the unbounded domain. In the case where the volume 
rate of the material is fixed and so is the depth of the walls, we expect that a large inclination angle will force all of the material to be in a flowing state.

As far as the boundary conditions are concerned, one could study the problem with friction both on a finite bottom of the inclined plane and on the walls. Moreover, it would be more realistic to include more general constitutive relations. But even the more general case of the $\mu(I)$-rheology, mentioned in the introduction presents instabilities, which suggests that there are physics missing in the model, as observed in [1]. Nevertheless, we hope that our rigorous and analytical study of our problem with Drucker-Prager plasticity, will facilitate the study of more complicated models.

\section{Appendix A. Regularity of minimizers of $E_{\varepsilon, \lambda}^{A}$}

In what follows we will denote by $c$ a generic constant which does not depend on the $\varepsilon$ mentioned in Proposition 3.4.

\section{Proof of Proposition 3.4}

Step 1. Existence/Uniqueness.

The uniqueness of the minimizer follows by the strict convexity of the functional or using similar arguments as in the proof of Step 3 of Theorem 2.2 (i). The existence is also similar, in fact the lower semicontinuity of the linear term $-\lambda \int_{\Omega_{A}} u$ is trivial since the domain $\Omega_{A}$ is bounded. We set

$$
F(z, p):=\frac{|p|^{2}}{2}+|z| \sqrt{\varepsilon^{2}+|p|^{2}},
$$

for $(z, p) \in \Omega_{A} \times \mathbb{R}^{2}$. It is

$$
|\xi|^{2} \leq \frac{\partial^{2} F}{\partial p_{i} \partial p_{j}} \xi_{i} \xi_{j}
$$

for $\xi=\left(\xi_{1}, \xi_{2}\right) \in \mathbb{R}^{2}$, and

$$
\left|\frac{\partial^{2} F}{\partial p_{i} \partial p_{j}}\right| \leq c\left(1+\frac{A}{\varepsilon}\right), \text { for all } i, j \in\{1,2\}
$$

we set $c_{2}:=c\left(1+\frac{A}{\varepsilon}\right)$.

Step 2. Regularity.

Since the proof of regularity is standard we are only going to emphasize the particularities of the problem, i.e. the fact that $F$ is only Lipschitz continuous in the $z$ variable. We will simply write $F(z, \nabla u)$ for $F(z, \nabla u(y, z))$. Let $\varphi$ with $\hat{\varphi} \in W_{0}^{1,2}\left(\hat{\Omega}_{A}\right)$, then equation (3.35) holds as the first variation of the functional $E_{\varepsilon, \lambda}^{A}$. Moreover, using a change of variables one can see that the function $w:=\hat{u}_{\varepsilon, A}$ satisfies

$$
\int_{\hat{\Omega}_{A}} \nabla w \cdot \nabla \varphi+|z| \frac{\nabla w \cdot \nabla \varphi}{\sqrt{\varepsilon^{2}+|\nabla w|^{2}}}=\lambda \int_{\hat{\Omega}_{A}} \varphi
$$

We study the regularity properties of (A.4). Let $|h|<\operatorname{dist}\left(\operatorname{supp} \varphi, \partial \hat{\Omega}_{A}\right)$, we define $\varphi_{k, h}(y, z):=\varphi((y, z)-$ $\left.h e_{k}\right), k=1,2$, with $e_{k}, k=1,2$ the unit vectors on the axes $y$ and $z$ respectively. We use $\varphi_{k, h}$ as a test function in (A.4) and estimate the derivative of the difference quotient

$$
\Delta_{h}^{k} w(y, z)=\frac{w\left((y, z)+h e_{k}\right)-w(y, z)}{h} .
$$


Since the proof is similar we will only present the estimate for $e_{2}$. Using $\varphi_{2, h}=\varphi_{h}$ as a test function in (A.4) and after changing the variables in the integral we get

$$
\int_{\hat{\Omega}_{A}} \partial_{p_{i}} F\left(z+h,(\nabla w)_{h}\right) \partial_{x_{i}} \varphi=\lambda \int_{\hat{\Omega}_{A}} \varphi
$$

where $\partial_{p_{i}} F=\frac{\partial F}{\partial p_{i}},(\nabla w)_{h}(y, z)=\nabla w(y, z+h)$ and $\partial_{x_{i}} \varphi, i=1,2$ is the partial derivative of $\varphi$ in the directions $y, z$ respectively. As usual, subtracting (A.4) from (A.6) we get after a few calculations

$$
\int_{\hat{\Omega}_{A}} \frac{1}{h}\left(\partial_{p_{i}} F\left(z+h,(\nabla w)_{h}\right)-\partial_{p_{i}} F(z+h, \nabla w)\right) \partial_{x_{i}} \varphi=-\int_{\hat{\Omega}_{A}} \frac{1}{h}\left(\partial_{p_{i}} F(z+h, \nabla w)-\partial_{p_{i}} F(z, \nabla w)\right) \partial_{x_{i}} \varphi .
$$

The right hand side of (A.7) can be estimated using the Lipschitz continuity of $\nabla_{p} F$ in the $z$ variable, we have

$$
\frac{1}{|h|}\left|\nabla_{p} F(z+h, \nabla w)-\nabla_{p} F(z, \nabla w)\right|=\frac{|\nabla w|}{\sqrt{\varepsilon^{2}+|\nabla w|^{2}}} \frac{|| z+h|-| z||}{|h|} \leq 1 .
$$

It is now a standard process to use (A.2) and (A.3) in order to bound the quantity $\int\left|\nabla \Delta_{h} w\right|^{2}$ uniformly in $h$, we have

$$
\int_{\Omega^{\prime}}\left|\nabla \Delta_{h} w\right|^{2} \leq 2 c_{3}\left(1+2 c_{2}\right) \int_{\Omega_{A}}|\nabla w|^{2}
$$

with $c_{3}$ a constant independent of $h$ and $\Omega^{\prime} \subset \subset \Omega^{\prime \prime} \subset \subset \Omega_{A}$. We then have $w \in W^{2,2}\left(\Omega^{\prime \prime}\right)$ by standard arguments.

Step 3. Neumann condition.

Since $\hat{u}_{\varepsilon} \in W_{\text {loc }}^{2,2}\left(\hat{\Omega}_{A}\right)$ we can define $\partial_{z} u_{\varepsilon}(y, 0)$ for a.e. $y \in(-1,1)$ and since $\hat{u}$ is symmetric with respect to $\{z=0\}$, it is in fact $\partial_{z} u_{\varepsilon}(y, z)=-\partial_{z} u_{\varepsilon}(y,-z)$ for $(y, z) \in \hat{\Omega}_{A}$; setting $z=0$ we get the desired result.

The constant $c_{2}$ in the estimate (A.8) depends on $\varepsilon$. Using an argument similar to the proof of [8], Theorem 3.3.4, we can show that the second derivative of $u_{\varepsilon}$ is bounded in $L^{2}$, uniformly in $\varepsilon$. We have the following lemma.

Lemma A.1. (Uniform bound on $\left|\nabla^{2} u_{\varepsilon}\right|$ )

Let $A>0, u_{\varepsilon}$ be as in Proposition 3.4 and $\Omega^{\prime} \subset \subset \hat{\Omega}_{A}$. Then there exists a positive constant $C=$ $C\left(A, \operatorname{dist}\left(\Omega^{\prime}, \partial \hat{\Omega}_{A}\right)\right)$ such that

$$
\int_{\Omega^{\prime}}\left|\nabla \partial_{x_{i}} \hat{u}_{\varepsilon}\right|^{2} \leq C\left(1+\int_{\hat{\Omega}_{A}}\left|\partial_{x_{i}} \hat{u}_{\varepsilon}\right|^{2}\right), \quad i=1,2
$$

\section{Proof of Lemma A.1}

Since the proof is similar to the proof of Proposition 3.4, we will only give a sketch of it. We will only show the proof of the estimate (A.9) for $\left|\nabla \partial_{z} \hat{u}_{\varepsilon}\right|$ because the term with the partial derivative in the $y$ variable is easier to estimate, since the integrand $F$ from (A.1) does not depend on $y$. Let $\varphi$ be a smooth function with compact support in $\Omega^{\prime \prime}$, with $\Omega^{\prime} \subset \subset \Omega^{\prime \prime} \subset \subset \Omega$; using $\partial_{z} \varphi$ as a test function in (A.4) and integrating by parts we can write, using the usual summation convention and the same notation as in the proof of Proposition 3.4

$$
\int_{\Omega^{\prime \prime}} \partial_{p_{i}} \partial_{z} F\left(z, \nabla \hat{u}_{\varepsilon}\right) \partial_{x_{i}} \varphi=0
$$


Noticing that $\partial_{z}\left(F\left(z, \nabla \hat{u}_{\varepsilon}(y, z)\right)\right)=\left.\partial_{\bar{z}} F\left(\bar{z}, \nabla \hat{u}_{\varepsilon}(y, z)\right)\right|_{\bar{z}=z}+\partial_{p_{j}} F\left(z, \nabla \hat{u}_{\varepsilon}(y, z)\right) \partial_{x_{j}} \partial_{z} \hat{u}_{\varepsilon}(y, z)$ and $\partial_{z}|z|=$ $\chi_{(0,+\infty)}-\chi_{(-\infty, 0)}$; we may rewrite (A.10) as

$$
\int_{\Omega^{\prime \prime}} \partial_{z}|z| \frac{\partial_{x_{i}} \hat{u}_{\varepsilon}}{\sqrt{\varepsilon^{2}+\left|\nabla \hat{u}_{\varepsilon}\right|^{2}}} \partial_{x_{i}} \varphi+\int_{\Omega^{\prime \prime}}\left(\partial_{p_{i}} \partial_{p_{j}} F\left(z, \nabla \hat{u}_{\varepsilon}\right) \partial_{x_{j}} \partial_{z} \hat{u}_{\varepsilon}\right) \partial_{x_{i}} \varphi=0
$$

As usual, we choose a function $\eta \in C_{0}^{2}\left(\Omega^{\prime \prime}\right)$ with $\eta=1$ in $\Omega^{\prime}, 0 \leq \eta \leq 1,|\nabla \eta| \leq \frac{c}{\operatorname{dist}\left(\Omega^{\prime}, \partial \Omega_{A}\right)}$ and $\left\|\nabla^{2} \eta\right\| \leq$ $\frac{c}{\left(\operatorname{dist}\left(\Omega^{\prime}, \partial \Omega_{A}\right)^{2}\right.}$. We set $\varphi:=\eta^{3} \partial_{z} \hat{u}_{\varepsilon}$ in (A.11), use the convexity property (A.2) and the fact that

$$
\partial_{p_{i}} \partial_{p_{j}} F\left(z, \nabla \hat{u}_{\varepsilon}\right) \partial_{x_{j}} \partial_{z} \hat{u}_{\varepsilon}=\partial_{x_{i}} \partial_{z} \hat{u}_{\varepsilon}+|z| \partial_{z}\left(\frac{\partial_{x_{i}} \hat{u}_{\varepsilon}}{\sqrt{\varepsilon^{2}+\left|\nabla \hat{u}_{\varepsilon}\right|^{2}}}\right),
$$

we get as in the proof of Proposition 3.4

$$
\begin{aligned}
\int_{\Omega^{\prime \prime}} \eta^{3}\left|\nabla \partial_{z} \hat{u}_{\varepsilon}\right|^{2} \leq & -\int_{\Omega^{\prime \prime}} \partial_{x_{i}} \partial_{z} \hat{u}_{\varepsilon} \partial_{x_{i}}\left(\eta^{3}\right) \partial_{z} \hat{u}_{\varepsilon}-\int_{\Omega^{\prime \prime}} \partial_{z}|z| \partial_{x_{i}}\left(\eta^{3}\right) \frac{\partial_{x_{i}} \hat{u}_{\varepsilon}}{\sqrt{\varepsilon^{2}+\left|\nabla \hat{u}_{\varepsilon}\right|^{2}}} \partial_{z} \hat{u}_{\varepsilon} \\
& -\int_{\Omega^{\prime \prime}} \partial_{z}|z| \eta^{3} \frac{\partial_{x_{i}} \hat{u}_{\varepsilon}}{\sqrt{\varepsilon^{2}+\left|\nabla \hat{u}_{\varepsilon}\right|^{2}}} \partial_{x_{i}} \partial_{z} \hat{u}_{\varepsilon}-\int_{\Omega^{\prime \prime}}|z| \partial_{z}\left(\frac{\partial_{x_{i}} \hat{u}_{\varepsilon}}{\sqrt{\varepsilon^{2}+\left|\nabla \hat{u}_{\varepsilon}\right|^{2}}}\right) \partial_{x_{i}}\left(\eta^{3}\right) \partial_{z} \hat{u}_{\varepsilon} \cdot(
\end{aligned}
$$

The first three terms of the right hand side of (A.12) can be estimated as in the proof of Proposition 3.4 using Young's inequality, the fact that $\partial_{z}|z| \leq 1$ and $\frac{\left|\partial_{x_{i}} \hat{u}_{\varepsilon}\right|}{\sqrt{\varepsilon^{2}+\left|\nabla \hat{u}_{\varepsilon}\right|^{2}}} \leq 1$, for $i=1,2$ uniformly in $\varepsilon$. We will only show the estimate of the last term of (A.12), which we denote by $J$. Integrating by parts $J$ we get

$$
\begin{aligned}
J= & \int_{\Omega^{\prime \prime}} \partial_{z}|z| \frac{\partial_{x_{i}} \hat{u}_{\varepsilon}}{\sqrt{\varepsilon^{2}+\left|\nabla \hat{u}_{\varepsilon}\right|^{2}}} \partial_{x_{i}}\left(\eta^{3}\right) \partial_{z} \hat{u}_{\varepsilon}+\int_{\Omega^{\prime \prime}}|z| \frac{\partial_{x_{i}} \hat{u}_{\varepsilon}}{\sqrt{\varepsilon^{2}+\left|\nabla \hat{u}_{\varepsilon}\right|^{2}}} \partial_{z} \partial_{x_{i}}\left(\eta^{3}\right) \partial_{z} \hat{u}_{\varepsilon} \\
& +\int_{\Omega^{\prime \prime}}|z| \frac{\partial_{x_{i}} \hat{u}_{\varepsilon}}{\sqrt{\varepsilon^{2}+\left|\nabla \hat{u}_{\varepsilon}\right|^{2}}} \partial_{z}\left(\eta^{3}\right) \partial_{z} \partial_{z} \hat{u}_{\varepsilon} .
\end{aligned}
$$

It is a standard process now to estimate the right hand side of the above equality using Young's inequality with weight $\gamma>0$, for example the last term of (A.13) can be estimated from above by

$$
c \int_{\Omega^{\prime \prime}} \eta^{1 / 2} \eta^{3 / 2}\left|\nabla \partial_{z} \hat{u}_{\varepsilon}\right| \leq \tilde{c}\left(\frac{1}{\gamma}+\gamma \int_{\Omega^{\prime \prime}} \eta^{3}\left|\nabla \partial_{z} \hat{u}_{\varepsilon}\right|^{2}\right)
$$

Finally, putting all the estimates together and choosing $\gamma$ small enough we can absorb the terms $\gamma \int_{\Omega^{\prime \prime}} \eta^{3}\left|\nabla \partial_{z} \hat{u}_{\varepsilon}\right|^{2}$ on the right hand side of (A.12) by its left hand side and by noticing that $\eta=1$ on $\Omega^{\prime}$ we end up with the desired estimate.

Acknowledgements. The authors would like to thank Professor François Bouchut for the valuable suggestions and the fruitful discussions concerning the connections of the mathematical model with the physical properties. They would also like to thank Professor Alexandre Ern for useful comments on the physical aspects of the problem, Professor Marco Cannone for his helpful suggestions on the presentation of this article and Dr. John Konstantinides for improving the quality of the three dimensional pictures. Lastly, the authors are thankful to the referees for the useful suggestions on the presentation of the paper and one of the referees for bringing to their attention the paper [16]. 


\section{REFERENCES}

[1] T. Barker, D.G. Schaeffer, P. Bohorquez and J.M.N.T. Gray, Well-posed and ill-posed behavior of the $\mu(I)$-rheology for granular flow. J. Fluid Mech. 779 (2015) 794-818.

[2] G. Bellettini, V. Caselles and M. Novaga, The total variation flow in $\mathbb{R}^{N}$. J. Differ. Equ. $184(2002) 475-525$.

[3] F. Bouchut, R. Eymard and A. Prignet, Convergence of conforming approximations for inviscid incompressible Bingham fluid flows and related problems. J. Evol. Equ. 14 (2014) 635-669.

[4] F. Bouchut, I.R. Ionescu and A. Mangeney, An analytic approach for the evolution of the static/flowing interface in viscoplastic granular flows. Commun. Math. Sci. 14 (2016) 14.

[5] O. Cazacu and I.R. Ionescu, Compressible rigid viscoplastic fluids. J. Mech. Phys. Solids 54 (2006) 1640 -1667

[6] G. Duvaut and J.-L. Lions, Inequalities in mechanics and physics. Translated from the French by C. W. John. Grundlehren der Mathematischen Wissenschaften. Springer-Verlag, Berlin, New York (1976).

[7] L.C. Evans, Partial differential equations, 2nd edn. Vol. 19 of Graduate Studies in Mathematics. American Mathematical Society, Providence, RI, (2010).

[8] M. Fuchs and G. Seregin, Variational methods for problems from plasticity theory and for generalized Newtonian fluids. Vol. 1749 of Lecture Notes in Mathematics. Springer-Verlag, Berlin (2000).

[9] M. Giaquinta, Multiple integrals in the calculus of variations and nonlinear elliptic systems. Vol. 105 of Annals of Mathematics Studies. Princeton University Press, Princeton, NJ (1983).

[10] M.-H. Giga, Y. Giga and N. Požár, Periodic total variation flow of non-divergence type in $\mathbb{R}^{n}$. J. Math. Pures Appl. 102 (2014) 203-233.

[11] Y. Giga and N. Požár, A level set crystalline mean curvature flow of surfaces. Adv. Differ. Equ. 21 (2016) 631-698.

[12] I.R. Ionescu, A. Mangeney, F. Bouchut and O. Roche, Viscoplastic modeling of granular column collapse with pressuredependent rheology. J. Non-Newton. Fluid Mech. 219 (2015) 1-18.

[13] P. Jop, Y. Forterre and O. Pouliquen, A constitutive law for dense granular flows. Nature 441 (2006) 727-730.

[14] D. Krejčiřík and A. Pratelli, The Cheeger constant of curved strips. Pac. J. Math. 254 (2011) 309-333.

[15] F. Krügel, Some properties of minimizers of a variational problem involving the total variation functional. Commun. Pure Appl. Anal. 14 (2015) 341-360

[16] F. Krügel, Potential theory for the sum of the 1-Laplacian and p-Laplacian. Nonlinear Anal. 112 (2015) $165-180$.

[17] G.P. Leonardi and A. Pratelli, On the Cheeger sets in strips and non-convex domains. Calc. Var. Partial Differ. Equ. 55 (2016) 15.

[18] G. Leoni, A first course in Sobolev spaces. Vol. 105 of Graduate Studies in Mathematics. American Mathematical Society, Providence, RI (2009).

[19] C. Lusso, F. Bouchut, A. Ern, A. Mangeney. A Free Interface Model for Static/Flowing Dynamics in Thin-Layer Flows of Granular Materials with Yield: Simple Shear Simulations and Comparison with Experiments. Appl. Sci. 7 (2017) 386.

[20] C. Lusso, A. Ern, F. Bouchut, A. Mangeney, M. Farin and O. Roche. Two-dimensional simulation by regularization of free surface viscoplastic flows with Drucker-Prager yield stress and application to granular collapse. J. Comput. Phys. 333 (2017) $387-408$.

[21] J. Málek, J. Nečas, M. Rokyta and M. Růžička. Weak and measure-valued solutions to evolutionary PDEs. Vol. 13 of Applied Mathematics and Mathematical Computation. Chapman \& Hall, London (1996).

[22] O. Pouliquen, C. Cassar, P. Jop, Y. Forterre and M. Nicolas. Flow of dense granular material: towards simple constitutive laws. J. Stat. Mech.: Theo. Exp. 7 (2006) P07020.

[23] G. Seregin, Continuity for the strain velocity tensor in two-dimensional variational problems from the theory of the Bingham fluid. Ital. J. Pure Appl. Math. 2 (1998) 141-150.

[24] D.G Schaeffer, Instability in the evolution equations describing incompressible granular flow. J. Differ. Equ. 66 (1987) 19-50.

[25] F.A. Vaillo, V. Caselles and J. M. Mazón, Parabolic Quasilinear Equations Minimizing Linear Growth Functionals. Vol. 223 of Progress in Mathematics. Birkhäuser Verlag, Basel (2004).

[26] W.P. Ziemer, Weakly Differentiable Functions. Vol. 120 of Graduate Texts in Mathematics. Springer-Verlag, New York (1989). 